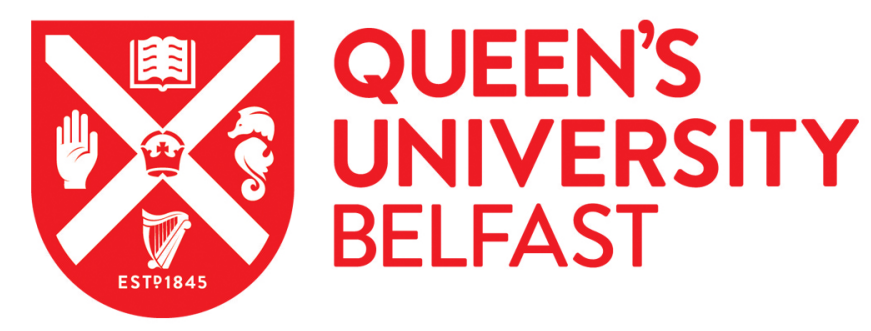

\title{
Popular Matching for Security-Enhanced Resource Allocation in Social Internet of Flying Things
}

Wang, B., Sun, Y., Duong, T. Q., Nguyen, L. D., \& Zhou, N. (2020). Popular Matching for Security-Enhanced Resource Allocation in Social Internet of Flying Things. IEEE Transactions on Communications, 68(8), 5087 5101. https://doi.org/10.1109/TCOMM.2020.2995223

\section{Published in:}

IEEE Transactions on Communications

\section{Document Version:}

Peer reviewed version

Queen's University Belfast - Research Portal:

Link to publication record in Queen's University Belfast Research Portal

Publisher rights

Copyright 2020 IEEE. This work is made available online in accordance with the publisher's policies. Please refer to any applicable terms of use of the publisher.

\section{General rights}

Copyright for the publications made accessible via the Queen's University Belfast Research Portal is retained by the author(s) and / or other copyright owners and it is a condition of accessing these publications that users recognise and abide by the legal requirements associated with these rights.

Take down policy

The Research Portal is Queen's institutional repository that provides access to Queen's research output. Every effort has been made to ensure that content in the Research Portal does not infringe any person's rights, or applicable UK laws. If you discover content in the Research Portal that you believe breaches copyright or violates any law, please contact openaccess@qub.ac.uk. 


\title{
Popular Matching for Security-Enhanced Resource Allocation in Social Internet of Flying Things
}

\author{
Bowen Wang, Yanjing Sun, Member, IEEE, Trung Q. Duong, Senior Member, IEEE, Long D. Nguyen, Nan \\ Zhao, Senior Member, IEEE
}

\begin{abstract}
As the Internet of Things (IoT) is maturing and acquires its social flavor, the Social IoT enables smart devices to build inter-thing social networks without human intervention. As a new form of smart devices, unmanned aerial vehicles (UAVs) are finding their way into IoT applications. The integrated Social Internet of Flying Things (SIoFT) can provide the socialaware UAV-assisted services. However, the broadcast nature of air-to-ground (A2G) channels makes them vulnerable to being eavesdropped by terrestrial malicious users due to their strong line-of-sight (LoS) links. In this paper, we investigate to ensure the security of A2G communications when the location information of multiple potential eavesdroppers cannot be perfectly estimated. Following the "no pain no gain" principle, the terrestrial users who reuse the UAV cellular spectrum will act as friendly jammers to realize "win-win" situation. Hence, joint trajectory design, power control, and channel allocation optimization problem is formulated to maximize the average secrecy rate of UAVs in worst case. In the first stage, we utilize the block coordinate descent method and successive convex optimization method to solve the trajectory design and power control problems in an iterative manner. In the second stage, we convert the user pairing problem into a popular matching problem with externalities. Two distributed algorithms are proposed to maintain the popular matching under dynamics. Moreover, we conduct detailed analysis of the popularity, convergence, and computational complexity. Simulation results demonstrate the superiority of our proposed method in terms of different performance metrics.
\end{abstract}

Index Terms-Social Internet of Flying Things, resource allocation, security, matching theory, graph theory.

\section{INTRODUCTION}

This work was supported in part by the Program for "Industrial IoT and Emergency Collaboration "Innovative Research Team in China University of Mining and Technology (CUMT) under Grant 2020ZY002; in part by the Key Research and Development Project for Science and Technology of Xuzhou, China, under Grant KC18105; and in part by the National Natural Science Foundation of China under Grant 51734009, Grant 61771417, and Grant 51804304. The work of Trung Q. Duong was supported in part by the Royal Academy of Engineering (RAEng) through the RAEng Research Fellowships schemer under Grant RF1415 \14 22 , and in part by the Researcher Links through the Newton Fund Partnership under Grant 527612186. The grant is funded by the U.K. Department for Business, Energy and Industrial Strategy and delivered by the British Council, for further information, please visit www.newtonfund.ac.uk

Bowen Wang and Yanjing Sun are with the School of Information and Control Engineering, China University of Mining and Technology, Xuzhou, 221116, China (e-mail: \{bowenwang, yjsun\}@cumt.edu.cn). Bowen Wang and Yanjing Sun are also with the Xuzhou Engineering Research Center of Intelligent Industry Safety and Emergency Collaboration, Xuzhou, 221116, China.

Trung Q. Duong are with the School of Electronics, Electrical Engineering and Computer Science, Queen's University, Belfast, BT7 1NN, UK (e-mail: trung.q.duong@qub.ac.uk).

Long D. Nguyen is with the Duy Tan University, Vietnam (e-mail: nguyendinhlong1@duytan.edu.vn).

Nan Zhao is with the School of Information and Communication Engineering, Dalian University of Technology, Dalian, 116024, China (e-mail: zhaonan@dlut.edu.cn).
$\mathbf{R}$ ECENT years have witnessed the continuous evolution of the Internet of Things (IoT), especially when the wave of $5 \mathrm{G}$ ecosystem is surging forward [1]. The IoT paradigm aims at revolutionizing the information interaction and perception ways of human beings in their daily life, by enabling automations of smart objects. IoT devices such as smart phones, home appliances, and wearable devices can autonomously make decisions, giving rise to the concept of Social IoT (SIoT) [2]. In line with the SIoT paradigm, the dramatically improved autonomous, computational, storage, and sensing capacities accelerate the adoption process of IoT devices. These smart devices can construct their social networks without human intervention and interact socially with each other.

Benefiting from the rapid development on electronic, sensor and communication technologies, the unmanned aerial vehicles (UAVs) are finding their way into civilian and military fields. The Flying Ad Hoc Networks (FANETs) paradigm has emerged by exploring the cooperation and collaboration between the UAVs [3]. Recently, some researchers have started to investigate the integration of FANETs into the IoT, which has led to the Internet of Flying Things (IoFT) [4]. The emergent IoFT aims at bringing the potential new degree of freedom into IoT, by utilizing the flexible mobility and deployment of UAVs. For example, UAVs can potentially replace the terrestrial IoT sensors thanks to their: 1) deployability in different landforms, 2) capability of carrying flexible payloads, and 3) programmability in mission [5]. However, the integration of UAVs into IoT also brings new challenges regarding security. Different from ground-to-ground (G2G) links, air-to-ground (A2G) links are generally dominated by line-of-sight (LoS) channels [6]. Although the strong LoS links can achieve high achievable rates, they are more vulnerable than $\mathrm{G} 2 \mathrm{G}$ links to eavesdropping and jamming attacks by terrestrial malicious users [7]. On the one hand, the reception quality of the terrestrial eavesdroppers is enhanced by exploiting the strong A2G LoS link and even a remote eavesdropper can overhear the A2G transmission clearly. On the other hand, a terrestrial jammer can also launch more effective attacks to UAVs by exploiting the strong ground-to-air (G2A) LoS links.

As a powerful method, information-theoretic physical layer security can maximize the reliable communication rate between two legitimate UAVs while ensuring that malicious users may learn as little information as possible [7]. However, this method ensures physical layer security at the cost of decreasing effective transmission rate, since all users are assumed to be not trustworthy [8], [9]. Hence, it is difficult to trade off between the security and throughput performances by solely resorting to physical layer security methods.

Based on the discussions above, a question thus arises: can 
we leverage the trustworthiness in social domain to ensure the security while reducing the sacrifice of achievable rate in the physical domain? To verify the influence of social trust on the physical layer security, the authors in [9] provided theoretical and numerical analysis of social trust aided cellular spectrum sharing via stochastic geometry. They demonstrated that the system ergodic rate can be increased by $63 \%$ on average based on a realistic dataset when the number of D2D pairs increases from 4 to 20 and the corresponding social link probability decreases from $70 \%$ to $25 \%$. Assuming that those users without social relations belong to the eavesdropper category, the authors in [10] utilized social-awareness to identify trusted and non-trusted users in device-to-device (D2D) underlaying cellular networks. In [11] and [12], the authors leveraged social-awareness to identify trusted and non-trusted users in relay selection scheme. All these studies verified the feasibility of exploiting the inter-human social ties to improve transmission security in G2G communications. Considering that UAVs usually perform missions in swarms, it is natural to exploit their social-awareness to further strengthen cooperation and collaboration between the UAVs. If the UAVs only share spectrum with their socially trusted devices, the number of potential eavesdroppers and jammers will be certainly reduced, and those trusted devices can act as friendly jammers to further improve the security. However, there are no works synthesizing the ideas from SIoT with those from the IoFT. In this paper, we first propose the concept of Social Internet of Flying Things (SIoFT), by integrating the social-awareness of UAVs into IoFT. However, the integrated SIoFT also poses some new challenges to the resource allocation. On the one hand, UAVs will balance the enhanced security performance and the reduced achievable rate performance when sharing spectrum with those trusted D2D users. On the other hand, the D2D users also want to increase their own utilities by avoiding the severe interference of strong A2G LoS links. It is challenging to design an effective interference management method to allocate spectrum resource for both aerial devices and terrestrial devices in a self-organizing manner.

\section{A. Related Works}

Recently, several research works have been rigorously studied to safeguard wireless communications with UAVs from a physical layer security perspective [13]-[15]. In [13], the authors investigated to maximize the average secrecy rate in a given period by joint UAV trajectory design and transmit power control. However, it is non-practical and optimistic to assume that the location information of the eavesdropper is perfectly recognized and obtained. In [14], assuming that the location information of an eavesdropper can be partially known by the camera or synthetic aperture radar equipped on an UAV, the authors proposed a jointly trajectory design and power control scheme to optimize the average worst case secrecy rate of UAV-to-ground communications. In [15], considering the unknown location information of the eavesdropper, the authors designed an altitude optimization method to maximize the average secrecy rate in the worst case. However, the impact of both social trust and friendly jammer selection on the security performance has not been addressed.
Some previous works have studied the security performance enhancement of $\mathrm{G} 2 \mathrm{G}$ communications through rationally selecting the friendly jammer [16], [17]. In [16], the authors introduced D2D communications underlaying cellular networks to improve the system secrecy capacity, by formulating the friendly jammer selection problem as a bipartite graph matching problem and invoking the classic Kuhn-Munkres (KM) algorithm to obtain an optimal solution in a centralized way. To further improve the security performance in the D2D communication underlying heterogeneous networks (HetNets), the authors in [17] proposed a centralized algorithm to solve the joint subcarrier allocation and power control problem in an iterative manner. However, the centralized optimization methods require global information exchange and centralized computation, which yields significant overhead and complexity [18]. For our SIoFT scenario, the distributed resource allocation methods are needed since each UAV or D2D user has to make self-organizing decisions with local information. Several existing works have resorted to game theory and matching theory to design distributed resource allocation methods in different scenarios [9], [10], [19]-[22]. In [19], the authors formulated the relay selection problem in multiUAV networks as a coalition formation game, and studied the interaction between source UAVs and relay UAVs. Similarly, the authors in [10] also proposed a coalition formation game based method to solve the security-ensured spectrum sharing problem. Different from the game-theoretic solutions which focus on the interactions among different users, the matching theory is a powerful and special tool to solve the user pairing problems [18]. In [20], the authors formulated the single relay selection problem in FIoT as a one-to-one matching model, and the classical Gale-Shapley (GS) algorithm in [23] was invoked to find a stable matching, in which no agents can be better of by deviating to another choice. The stable matching can achieve the Pareto optimility. In [21], the authors formulated the spectrum sharing problem in SIoT as a manyto-one matching model with externalities, and proposed a rotation-swap algorithm to tackle the externalities caused by dynamically changing co-channel interference (i.e., the join or leave of those D2D users in the same spectrum reusing group will cause the dynamic changes of co-channel interference). Similarly, the authors in [9] formulated the secure resource allocation problem in social-aware D2D communications as a many-to-one matching model with externalities. However, the conventional stable matching model can be further extended to popular matching model, by shifting from the local stability to global stability.

In [24] and [25], the authors proposed the concept of popular matching in which a matching $\mathcal{M}$ is popular if there exits no matching where more agents are better off than in $\mathcal{M}$. They also proved that stable matching is equivalent to the minimum size popular matching and computing a popular matching is NP-hard. The authors in [26] investigated how to obtain a maximum size popular matching in one-to-one matching model with polynomial time complexity. Based on this algorithm, the authors in [22] designed an effective algorithm to compute a maximum size popular in many-to-one matching model without externalities. The work in [22] is also the first to 
introduce popular matching to IoT scenario. However, all the above mentioned works on popular matching can only solve the matching problem without externalities, since preferences are assumed to be fixed. Note that externalities also exist in our scenario, since the preferences of D2D users is affected by not only UAVs, but also the join and leave of D2D users (i.e., both the trustworthiness and co-channel interference are dynamically changing), which inspires us to design an effective method.

\section{B. Main Contributions}

In this paper, to address the aforementioned challenges, we study the security-enhanced resource allocation problem in SIoFT, aiming at striking a trade-off between the average worst-case secrecy rate and the achievable achievable rate for both UAV communications and D2D communications. Our main contributions are listed as follows:

- Modeling: By integrating the social-awareness of UAVs into IoFT, we propose the concept of SIoFT, where UAVs can utilize social relations to identify trusted and nontrusted users. In this regard, we leverage the inter-device social ties to safeguard the security without sacrificing too much achievable rate in the physical domain, where there are multiple eavesdroppers and only partial location information can be obtained.

- Problem Formation: The optimization problem is intractable due to its non-convexity and semi-infinite number of constraints. Considering the mixed continuous variables (the coordinates and power of UAVs) and discrete variable (the binary variable for channel allocation), we decouple the original problem into two sub-problems: 1) the joint trajectory design and power control subproblem; and 2) the channel allocation sub-problem between UAVs and D2D users. The first sub-problem can be formulated as a semidefinite programming problem based on S-Procedure, while the second sub-problem is converted to a many-to-one popular matching problem with externalities.

- Algorithm Design: In the first stage, we utilize block coordinate descent method and successive convex optimization method to solve the first sub-problem in an iterative way. In the second stage, we propose two tailored algorithms to solve the formulated popular matching problem. In particular, we propose a hierarchical matching algorithm to initially construct the maximum popular matching structure as far as possible. Note that this matching may not be popular, since the preference profiles of some D2D users will change in the matching phase. To tackle this problem, we propose a rotation-vote algorithm to maintain the popularity under externalities.

- Validations: We comprehensively prove the popularity, convergence, and complexity properties of our proposed method. Under various scenarios, simulation results demonstrate that popular matching model based method outperforms the classical stable matching based method in terms of both security and achievable rate performances.

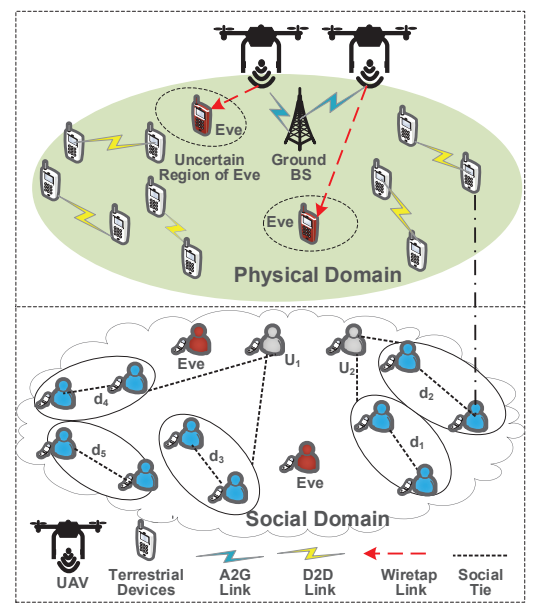

Fig. 1: Framework of SIoFT.

The rest of this paper is outlined as follows. The framework for IoFT scenario is presented and then the optimization problem is formulated in Section II. The method to solve joint trajectory design and power control problem is illustrated in Section III. The popular matching based channel allocation is introduced in Section IV. Simulation results are shown in Section V, followed by concluding remarks in Section VI.

\section{System Model And Problem Formulation}

As shown in Fig.1, we consider a typical SIoFT framework in a time slotted system, in which the flight duration $\mathcal{T}$ is quantized into $T$ sufficiently small time slots with equal length $\Delta$, i.e., $\mathcal{T}=T \Delta$, and the set of cumulative slots is denoted by $\{1, \ldots, t, \ldots, T\}$. Since $\Delta$ is small enough, the network topology can be regarded as static within each slot. In this scenario, a set of UAVs $\mathcal{U}=\left\{u_{j}\right\}_{j=1}^{M}$ mounted with sensor, computing, and communication modules, can share their collected data with a common base station (BS), i.e., $b$, through cellular uplinks in each time slot. Generally, each UAV will fly straight to the location right above the BS, then hover there to maximize the transmission date, and finally fly to the final location at the end of its flight duration. However, the UAV-Ground communications dominated by strong LoS channels may be intercepted by a set of eavesdroppers $\mathcal{E}=\left\{e_{k}\right\}_{k=1}^{K}$ and each eavesdropper is assumed to operate at one sub-channel [17]. To tackle against the eavesdroppers, the UAVs can adjust their trajectory to bypass the eavesdroppers along the way, control their powers when flying near the eavesdroppers, and share the cellular spectrum with a set of D2D pairs $\mathcal{D}=\left\{d_{i}\right\}_{i=1}^{N}$, which can act as friendly jammers to safeguard the cellular uplink. Each D2D pair $d_{i}$ consists of one transmitter $d_{i}^{T r}$ and one receiver $d_{i}^{R e}$, and works in underlay mode. i.e., reusing the spectrum of cellular UAVs. However, some malicious D2D pairs can also create security risks. In [9], [10], the authors assumed that those D2D users without trustworthiness can be viewed as eavesdroppers, and then the cellular users can identify the trusted or non-trusted D2D users based on the inter-human social trust. Therefore, the cellular users and D2D users can choose to share spectrum with trust users to ensure the security. Besides, the social tie also provides incentive for D2D users to act as friendly 
jammers. The smart IoT devices acquire their social-awareness abilities and build their inter-device social networks, which conforms to the SIoT paradigm. By analogy, we assume that IoT devices can identify trusted or non-trusted users based on the inter-device social trust. Herein, we use a binary variable $s_{i, j}$ to determine whether or not two devices are socially connected. For example, in Fig.1, the D2D pair $d_{4}, d_{5}$ and the UAV $u_{2}$ have no social tie with each other, all of them will worry about the other's eavesdropping and jamming when sharing the same channel.

In this paper, we consider the orthogonal frequency division multiple access (OFDMA) scheme, where each UAV is equally allocated to one dedicated subchannel. Similar to [27], we assume that the local channel state information (CSI) can be obtained by conventional channel estimation techniques such as pilot signaling [28]. In the local CSI scenario, the source node, i.e., UAV, has the knowledge of the CSI to BS and D2D receivers. However, although the UAVs can roughly recognize the location of eavesdroppers, the location information suffers from large estimation errors since the eavesdroppers hide themselves by remaining silent. Similarly, each D2D pair has the knowledge of the CSI to its receiver, BS, and other D2D receivers reusing the same spectrum. Note that the above information can be exchanged in spectrum sharing stage [10], [27], [29]. Generally, we consider the 3D Cartesian coordinate system in which the euclidean distance between two devices' coordinates are used to denote their distance. For D2D user $d_{i}$, the real-time coordinates of transmitter $d_{i}^{T r}$ and receiver $d_{i}^{R e}$ are denoted as $\left(x_{i}^{T r}(t), y_{i}^{T r}(t), 0\right)$ and $\left(x_{i}^{R e}(t), y_{i}^{R e}(t), 0\right)$, respectively. Similarly, the coordinate of UAV $u_{j}$ is $\left(x_{j}(t), y_{j}(t), H\right){ }^{1}$ Generally, the eavesdropper may remain silent to hide its existence and thus the location estimation using the synthetic aperture radar is expected to suffer from errors, which makes the accurate CSI of the eavesdropper difficult to acquire. Similar to [14], assuming that the roughly estimated location is known, the imperfect CSI can be computed according to the estimation errors. Herein, the exact location of $e_{k}$ in time slot $t$, denoted by $\left(x_{k}(t), y_{k}(t), 0\right)$, cannot be exactly known, i.e., only the roughly estimated location $\left(x_{k}^{E}(t), y_{k}^{E}(t), 0\right)$ is known [14]. The relation between these two locations can be represented as [14]

$$
x_{k}(t)=x_{k}^{E}(t)+\Delta x_{k}, y_{k}(t)=y_{k}^{E}(t)+\Delta y_{k},
$$

where $\Delta x_{k}$ and $\Delta y_{k}$ denote the estimation errors, which meet the following condition [14]

$$
\Delta x_{k}^{2}+\Delta y_{k}^{2} \leq \mathcal{R}_{k}^{2},
$$

where $\mathcal{R}_{k}$ denotes the radius of the uncertain circular region.

For a typical UAV $u_{j}$, its trajectory set within a given duration $\mathcal{T}$ can be denoted as $\left\{\left(x_{j}(t), y_{j}(t), H\right)\right\}_{t=1}^{T}$. For two consecutive time slots, e.g., $t$ and $t+1$ with $0<t<T$, the mobility constraints can be given by

$$
\sqrt{\left(x_{j}(t+1)-x_{j}(t)\right)^{2}+\left(y_{j}(t+1)-y_{j}(t)\right)^{2}} \leq v_{\max } \Delta,
$$

\footnotetext{
${ }^{1}$ Similar to [14], each UAV is assumed to fly at a constant altitude for safety considerations such as building avoidance.
}

where $v_{\max }$ represents the maximum flying speed. Note that the initial location and final location for $u_{j}$ are $\left(x_{j}(0), y_{j}(0), H\right)$ and $\left(x_{j}(T+1), y_{j}(T+1), H\right)$, respectively, which can ensure that each UAV can fly from initial location to final location.

Based on the practical measurement results in [30], the A2G communications can be approximated by LoS channel model very well. Hence, the A2G channel from the UAV to the terrestrial device is assumed to be dominant by LoS link [14]. In time slot $t$, the channel gain from the UAV $u_{j}$ to the BS $b$ is expressed as

$$
\begin{aligned}
g_{j, b}(t) & =\eta_{0} d_{i, b}^{-\alpha}(t) \\
& =\frac{\eta_{0}}{\left(x_{j}(t)-x_{b}\right)^{2}+\left(y_{j}(t)-y_{b}\right)^{2}+H^{2}},
\end{aligned}
$$

where $\eta_{0}$ represents the power gain of a LoS channel with unit reference distance, i.e., $d_{0}=1 \mathrm{~m}$, and the path loss parameter $\alpha$ for A2G link is set to 2 [14].

Besides, we use the practical Rayleigh fading channel to model the regular D2D link [17]. The power gain from the D2D transmitter $d_{i}^{T r}$ to another device $d_{i}^{R e}$ is expressed as

$$
g_{d_{i}^{T}, d_{i}^{R}}(t)=d_{d_{i}^{T}, d_{i}^{R}}^{-\alpha_{G}}(t)\left|h_{d_{i}^{T}, d_{i}^{R}}\right|^{2},
$$

where $\alpha_{G}$ denotes the path loss exponents for G2G link and $h_{d_{i}^{T}, d_{i}^{R}}$ is the complex Gaussian channel coefficient which follows the distribution of $\mathcal{C N}(0,1)$.

We use $P_{j}(t), \bar{P}$, and $\hat{P}$ to denote the real-time transmit power, average power, and peak power of $u_{j}$, respectively. Then, the transmit power of UAV should satisfy the following conditions

$$
\begin{aligned}
& \frac{1}{T} \sum_{t=1}^{T} P_{j}(t) \leq \bar{P}, \forall u_{j} \in \mathcal{U}, \\
& 0 \leq P_{j}(t) \leq \hat{P}, \forall u_{j} \in \mathcal{U} .
\end{aligned}
$$

Similar to [14], we assume that $\bar{P}<\hat{P}$ to ensure that (6a) is a non-trivial constraint. The channel allocation decisions are denoted as a $N \times M \times T$ matrix $\boldsymbol{\omega}_{N \times M \times T}$. Here, the $(i, j, t)$ th $\omega_{N \times M \times T}$, i.e., $\omega_{i, j, t}$, is a binary variable, where $\omega_{i, j, t}=1$ or 0 can determine whether or not $d_{i}$ reuses $u_{j}$ 's spectrum resource in slot $t$. When the UAV $u_{j}$ shares its uplink spectrum resource with D2D user $d_{i}$, the real-time achievable rates at the ground $\mathrm{BS}\left(R_{j, b}\right), \mathrm{D} 2 \mathrm{D}$ receiver $\left(R_{i}\right)$, eavesdropper $e_{k}$ against $u_{j}\left(R_{j, k}\right)$, Eavesdropper $e_{k}$ against $d_{i}\left(R_{i, k}\right)$ in bps/Hz are

$$
\begin{gathered}
R_{j, b}(t)=\log _{2}\left(1+\frac{P_{j}(t) g_{j, b}(t)}{I_{i, b}^{j}(t)+N_{0}}\right), \\
R_{i}(t)=\log _{2}\left(1+\frac{P_{i} g_{d_{i}^{T}, d_{i}^{R}}(t)}{I_{j, i}(t)+I_{i^{\prime}, i}^{j}(t)+N_{0}}\right), \\
R_{j, k}(t)=\log _{2}\left(1+\frac{P_{j}(t) g_{j, k}(t)}{I_{i, k}^{j}+N_{0}}\right), \\
R_{i, k}(t)=\log _{2}\left(1+\frac{P_{i} g_{i, k}(t)}{I_{j, k}(t)+I_{i^{\prime}, k}^{j}(t)+N_{0}}\right)
\end{gathered}
$$


where $P_{i}$ is the fixed transmission power of the $d_{i}^{T r}$. $I_{i, b}^{j}(t)=\sum_{i=1}^{N} \omega_{i, j, t} P_{i} g_{i, j}(t), I_{j, i}(t)=P_{j}(t) g_{i, j}, I_{i^{\prime}, i}^{j}(t)=$ $\sum_{i^{\prime} \neq i} \omega_{i^{\prime}, j, t} P_{i^{\prime}} g_{i^{\prime}, i}(t), \quad I_{i, k}^{j}(t)=\sum_{i=1}^{N} \omega_{i, j, t} P_{i} g_{i, k}(t)$, $I_{j, k}(t)=P_{j}(t) g_{j, k}$, and $I_{i^{\prime}, k}^{j}=\sum_{i^{\prime} \neq i} \omega_{i^{\prime}, j, t} P_{i^{\prime}} g_{i^{\prime}, k}(t)$, represent the co-channel interference from those D2D users reusing $u_{j}$ 's channel to $\mathrm{BS} b$, from UAV $u_{j}$ to D2D user $d_{i}$, from those D2D users reusing $u_{j}$ 's channel except $d_{i}$ to UAV $u_{j}$, from those D2D users reusing $u_{j}$ 's channel to eavesdropper $e_{k}$, from UAV $u_{j}$ to eavesdropper $e_{k}$, from those D2D users reusing $u_{j}$ 's channel except $d_{i}$ to eavesdropper $e_{k}$, and from those D2D users reusing $u_{j}$ 's channel except $d_{i}$ to UAV $u_{j}$ in slot $t$, respectively. Note that we use binary variable $\omega_{i, j, t}$ defined before to determine whether or not one D2D user interferes with other D2D users in slot $t$. Similarly, $g_{j, k}(t), g_{i, k}(t)$, and $g_{i, i^{\prime}}(t)$ represent the path loss from UAV $u_{j}$ to eavesdropper $e_{k}$, from D2D user $d_{i}$ to eavesdropper $e_{k}$, and between two D2D users $d_{i}$ and $d_{i^{\prime}}$ in slot $t$, respectively. $N_{0}$ denotes the variance of Gaussian noise.

As discussed before, we allocate spectral resources to D2D users for their communication requirements, while these D2D users will act as friendly jammers by sacrificing part of security. Meanwhile, the resource providers (UAVs) will sacrifice part of achievable rates to enhance the information secrecy. Therefore, all devices follow the "no pain no gain" principle and achieve the "win-win" situation, i.e., the UAVs want to sacrifice part of achievable rates to enhance the secrecy rate while the D2D users want to reuse the UAVs' spectrum by acting as friendly jammers. As such, we leverage their socialawareness to identify trusted and non-trusted users. In [2], the inter-device social relationships in SIoT can be categorized as (i) ownership object relationship (OOR) which can be establish based on whether two objects belongs to the same owner, (ii) co-work object relationship (CWOR) which can be establish based on whether two objects perform the same task, (iii) colocation object relationship (CLOR) which can be establish based on whether two objects are in the same place, and (iv) social object relationship (SOR) which can be establish based on their owners' social relationships. Note that alll these relationships can be extracted through the interaction history, cooperation object, and real-time location. Since the UAVs also belong to the smart objects in IoT, inter-device social relationships in SIoT can also be applied into our scenario. For simplicity, we only use the binary variable $s$ defined before to determine whether or not the social trust exists between two devices rather than measure its strength, i.e., $s_{i, j}$ can refer to the social tie between UAV $u_{j}$ and D2D user $d_{i}$. Considering that these non-trusted users belong to the eavesdropper category [9], [10], UAV communications should be safeguarded against both the eavesdroppers and non-trusted D2D users sharing the same spectrum resource. Herein, we only consider the fixed social tie, since the social relationships change slowly over time [11].

In our scenario, we ensure the security of all the UAVs and D2D users. Assuming that the eavesdroppers cannot cooperate, the expressions of UAV $u_{j}$ 's average worst-case secrecy rate over the period $\mathcal{T}$ against eavesdroppers can be given by [14]

$$
\mathcal{C}_{s}^{\text {ave }}\left(u_{j}\right)=\frac{1}{T} \sum_{t=1}^{T}\left[R_{j, b}(t)-\max _{e_{k} \in \mathcal{E}} \max _{\Delta x_{k}^{2}+\Delta y_{k}^{2} \leq \mathcal{R}_{k}^{2}} R_{j, k}(t)\right]^{+},
$$

where $[x]^{+}=\max \{0, x\}$.

Besides, we also safeguard the information security of D2D users, and the real-time worst-case secrecy rate of $d_{i}$ against eavesdroppers can be denoted as

$$
\mathcal{C}_{s}^{r}\left(d_{i}, t\right)=\left[R_{i}(t)-\max _{e_{k} \in \mathcal{E}} \max _{\Delta x_{k}^{2}+\Delta y_{k}^{2} \leq \mathcal{R}_{k}^{2}} R_{i, k}(t)\right]^{+} .
$$

In this paper, each user is assumed to be selfish and rational, i.e., each user wants to improve its own utility [18]. Note that this assumption is not orthogonal to the defined social relationships, because the users can utilize the social relationships to benefit themselves and achieve the win-win sitation through cooperation. The objective of the UAVs is to maximize the average worst-case secrecy rate in (11) under the constraint of achievable rate, by jointly considering the trajectory design, power control, and channel allocation. The optimization variables include the location, power, and channel allocation matrix, which are denoted as $\boldsymbol{x}_{M \times T}, \boldsymbol{y}_{M \times T}$, $\boldsymbol{P}_{M \times T}$, and $\boldsymbol{\omega}_{N \times M \times T}$. The optimization problem can be derived as

$$
\begin{array}{cl} 
& \text { P1 } \max _{\boldsymbol{\omega}, \boldsymbol{x}, \boldsymbol{y}, \boldsymbol{P}} \frac{1}{M} \sum_{j=1}^{M} \mathcal{C}_{s}^{\text {ave }}\left(u_{j}\right) \\
\text { s.t. } & I_{i, b}^{j}(t) \leq I^{t h}, \forall u_{j} \in \mathcal{U}, \forall d_{i} \in \mathcal{D}, t \in \mathcal{T}, \\
& \sum_{j=1}^{M} \omega_{i, j, t} \leq 1, \sum_{i=1}^{N} \omega_{i, j, t} \leq q_{j}, \sum_{t=1}^{T} \omega_{i, j, t} \leq T, \\
& s_{i, j}=1, \forall \omega_{i, j, t}=1,(3),(6),
\end{array}
$$

where $I^{t h}$ denotes the mutual interference threshold of UAVs. Constraint (13c) ensures that each D2D user can reuse at most one UAV's spectrum in a given time slot, each UAV has a quota $q_{j}$ for spectrum sharing in a given time slot, and each D2D user can access at most $T$ slots. (13d) can ensure that each UAV only shares spectrum with its socially trusted users, while (3) and (6) defined before give its mobility and power constraints.

In addition, the objective of the D2D users is to maximize the achievable rate under the constraint of average worst-case secrecy rate. Herein, we assume that each D2D user can only utilize the local CSI to make decisions on spectrum sharing. The optimization problem can be given by

$$
\begin{gathered}
\text { P2 } \max _{\omega} \frac{1}{T N} \sum_{i=1}^{N} \sum_{t=1}^{T} R_{i}(t) \\
\text { s.t. } \quad \mathcal{C}_{s}^{r}\left(d_{i}, t\right) \geq \mathcal{C}_{s}^{t h}, \text { if } \sum_{j=1}^{M} \omega_{i, j, t}=1, \forall t, i, \\
\quad \sum_{j=1}^{M} \omega_{i, j, t} \leq 1, \sum_{i=1}^{N} \omega_{i, j, t} \leq q_{j}, \sum_{t=1}^{T} \omega_{i, j, t} \leq T,
\end{gathered}
$$

where $\mathcal{C}_{s}^{\text {th }}$ denotes the real-time worst-case secrecy rate threshold of D2D users. (14b) ensures that the real-time secrecy rates 
of those D2D users act as friendly jammers in slot $t$ should exceed a threshold. Note that all those D2D links have been already eatablished, and thus the constraint that each D2D transmitter can only be paired with one D2D receiver at the same time is implicit in both (13) and (14).

It is difficult to simultaneously solve problems (13) and (14) because of the following reasons: 1) the operator $[\cdot]^{+}$ introduces non-smoothness to the problem (13) whose objective function is still not jointly concave with respect to the optimization variables; 2) the infinite number of possible $\left(\Delta x_{k}, \Delta y_{k}\right)$ makes (13) an intractable semi-infinite optimization problem; and 3) solving the channel allocation problem for both (13) and (14) belongs to the category of NP-hard combinatorial optimization problem due to its binary variable $\omega$ [27]. Note that $\boldsymbol{x}, \boldsymbol{y}$, and $\boldsymbol{P}$ are continuous variables while $\boldsymbol{\omega}$ is a discrete variable. In the following sections, we first decouple the optimization problem into two sub-problems, which can be solved step by step.

\section{Joint Trajectory Design And POWER CONTROL}

In the section, we optimize problem (13) by solving joint trajectory design and power control problem in an iterative way. Firstly, we tackle the non-smoothness of the objective function of problem (13) by using the lemma in [14].

Lemma 1 [14]: Problem (13) shares the same optimal solution with the following problem:

$$
\max _{\boldsymbol{\omega}, \boldsymbol{x}, \boldsymbol{y}, \boldsymbol{P}} \frac{1}{M} \sum_{j=1}^{M} \sum_{t=1}^{T}\left[R_{j, b}(t)-\max _{e_{k} \in \mathcal{E}} \max _{\Delta x_{k}^{2}+\Delta y_{k}^{2} \leq \mathcal{R}_{k}^{2}} R_{j, k}(t)\right]
$$

s.t. $(13 b)-(13 d)$

Proof: Let $V_{1}^{*}$ and $V_{2}^{*}$ denote the optimal values of problem (13) and (15), respectively. Since $[V]^{+} \geq V, \forall V$, $V_{1}^{*} \geq V_{2}^{*}$. Let $\left(\boldsymbol{\omega}^{*}, \boldsymbol{x}^{*}, \boldsymbol{y}^{*}, \boldsymbol{P}^{*}\right)$ denote the optimal solution of problem (13) and $f(P(t))=R_{j, b}(t)-$ $\max _{e_{k} \in \mathcal{E}} \max _{\Delta x_{e_{k}}^{2}+\Delta y_{e_{k}}^{2} \leq \mathcal{R}_{e_{k}}} R_{j, k}(t)$. We further give a feasible solution $(\dot{\omega}, \dot{x}, \dot{y}, \dot{P})$, where $\dot{\omega}=\omega^{*}, \dot{x}=x^{*}, \dot{y}=y^{*}$. The elements of $\dot{P}$ satisfy the condition: if $f(P(t)) \geq 0$, $\dot{\boldsymbol{P}}=\boldsymbol{P}^{*}$; otherwise $\dot{\boldsymbol{P}}=0$. Let $\dot{V}$ denote the value obtained at $(\dot{\boldsymbol{\omega}}, \dot{\boldsymbol{x}}, \dot{\boldsymbol{y}}, \dot{\boldsymbol{P}})$ and the condition $\dot{V}=V_{1}^{*}$ is satisfied, which proves that $V_{1}^{*}=V_{2}^{*}$ since $V_{2}^{*} \geq \dot{V}=V_{1}^{*}$ and $V_{1}^{*} \geq V_{2}^{*}$.

The non-convexity of problem (15) makes it difficult to tackle. Through observation, the optimization variables can be first partitioned into two categories, i.e., $\boldsymbol{x}, \boldsymbol{y}$, and $\boldsymbol{P}$ are continuous variables while $\omega$ is a discrete variable. With the fixed discrete variable, the problem (15) becomes more tractable. In this section, we first optimize the problem (15) with the fixed $\omega$. In this way, only the constraints (3) and (6) are considered, because the others are not coupled with $\boldsymbol{x}, \boldsymbol{y}$, and $P$. In the next section, we further optimize this problem with given $(\boldsymbol{x}, \boldsymbol{y}, \boldsymbol{P})$. Note that only the decisions on $\boldsymbol{\omega}$ can affect each other and one UAV's decisions on $(\boldsymbol{x}, \boldsymbol{y}, \boldsymbol{P})$ have no effect on each other. If the $\omega$ is given, the problem (15) can be decoupled into $M$ individual sub-problems as follows

$$
\max _{\boldsymbol{x}_{j}, \boldsymbol{y}_{j}, \boldsymbol{P}_{j}} \sum_{t=1}^{T}\left[R_{j, b}(t)-\max _{e_{k} \in \mathcal{E}} \max _{\Delta x_{k}^{2}+\Delta y_{k}^{2} \leq \mathcal{R}_{k}^{2}} R_{j, k}(t)\right], \forall u_{j} \in \mathcal{U}
$$

$$
\text { s.t. }(3),(6) \text {, }
$$

where $\boldsymbol{x}_{j}=\left[x_{j}(1), \ldots, x_{j}(T)\right]^{\top}, \boldsymbol{y}_{j}=\left[y_{j}(1), \ldots, y_{j}(T)\right]^{\top}$, and $\boldsymbol{P}_{j}=\left[P_{j}(1), \ldots, P_{j}(T)\right]^{\top}$, where $[\cdot]^{\top}$ is the transpose operation. Similarly, when optimizing the problem (16) with continuous variables, we further partition these variables into two blocks, i.e., trajectory $\left(\boldsymbol{x}_{j}, \boldsymbol{y}_{j}\right)$ and power $\boldsymbol{P}_{j}$. Thus, we can optimize $\boldsymbol{P}_{j}$ with given $\left(\boldsymbol{x}_{j}, \boldsymbol{y}_{j}\right)$ and optimize $\left(\boldsymbol{x}_{j}, \boldsymbol{y}_{j}\right)$ with given $\boldsymbol{P}_{j}$ in an iterative manner.

\section{A. Power Control under Given Trajectory}

With the given trajectory $(\boldsymbol{x}, \boldsymbol{y})$, the problem (16) can be rewritten as

$$
\max _{\boldsymbol{P}_{j}} \sum_{t=1}^{T}\left[\log _{2}\left(1+\alpha(t) P_{j}(t)\right)-\log _{2}\left(1+\beta(t) P_{j}(t)\right)\right]
$$

$$
\text { s.t. }(6)
$$

where

$$
\begin{aligned}
& \alpha(t)=\frac{\gamma_{0}}{\left(x_{j}(t)-x_{b}\right)^{2}+\left(y_{j}(t)-y_{b}\right)^{2}+H^{2}}, \\
& \beta(t)=\frac{\gamma_{0}}{\min _{e_{k} \in \mathcal{E}} \theta_{k}(t)}, \theta_{k}(t)=\min _{\Delta x_{k}^{2}+\Delta y_{k}^{2} \leq \mathcal{R}_{k}^{2}} \\
& \left(x_{j}(t)-x_{k}(t)\right)^{2}+\left(y_{j}(t)-y_{k}(t)\right)^{2}+H^{2}
\end{aligned}
$$

$$
\gamma_{0}=\frac{\eta_{0}}{I_{i, b}^{j}(t)+N_{0}}
$$

By substituting (1) and (2) into (19), $\theta_{k}(t)$ can be simplified to

$$
\theta_{k}(t)= \begin{cases}H^{2}, & \text { if } d_{k}(t) \leq \mathcal{R}_{k} \\ \left(d_{k}(t)-\mathcal{R}_{k}\right)^{2}+H^{2}, & \text { otherwise }\end{cases}
$$

where $d_{k}(t)=\sqrt{\left.\left(x_{j}(t)-x_{k}(t)\right)^{2}+\left(y_{j}(t)-y_{k}(t)\right)^{2}\right)}$. Based on [14], the optimal solution of problem (17) can be given by

$$
P_{j}^{*}(t)= \begin{cases}\min \left(\left[\widetilde{P}_{j}\right]^{+}, \hat{P}\right), & \text { if } \alpha(t)>\beta(t), \\ 0, & \text { otherwise }\end{cases}
$$

where

$$
\begin{aligned}
\widetilde{P}_{j}= & \sqrt{\left(\frac{1}{2 \beta(t)}-\frac{1}{2 \alpha(t)}\right)^{2}+\frac{1}{\xi \ln 2}\left(\frac{1}{\beta(t)}-\frac{1}{\alpha(t)}\right)} \\
& -\frac{1}{2 \beta(t)}-\frac{1}{2 \alpha(t)} .
\end{aligned}
$$

Note that $\xi$ determined by bisection search [31] is a parameter to satisfy (6a). 


\section{B. Trajectory Design under Given Power}

Let $P_{j}=\eta_{0} P_{j}^{*}(t)$, the problem (16) can be rewritten as

$$
\begin{aligned}
& \max _{\boldsymbol{x}_{j}, \boldsymbol{y}_{j}} \sum_{t=1}^{T}\left[\log _{2}\left(1+\frac{P_{j}}{\left(x_{j}(t)-x_{b}\right)^{2}+\left(y_{j}(t)-y_{b}\right)^{2}+H^{2}}\right)\right. \\
& \left.-\log _{2}\left(1+\frac{P_{j}}{\min _{e_{k} \in \mathcal{E}} \min _{\Delta x_{k}^{2}+\Delta y_{k}^{2} \leq \mathcal{R}_{k}} f\left(x_{j}(t), y_{j}(t)\right)}\right)\right]
\end{aligned}
$$

s.t. $\quad(3)$

where $f\left(x_{j}(t), y_{j}(t)\right)=\left(x_{j}(t)-x_{k}(t)\right)^{2}+\left(y_{j}(t)-y_{k}(t)\right)^{2}+$ $H^{2}$.

Since the (24) is still non-convex, we introduce two slack variables $\boldsymbol{b}_{j}=\left[b_{j}(1), \ldots, b_{j}(T)\right]^{\top}$ and $\boldsymbol{c}_{j}=\left[c_{j}(1), \ldots, c_{j}(T)\right]^{\top}$ into (24), which can be equivalent as

$$
\begin{aligned}
& \max _{\boldsymbol{x}_{j}, \boldsymbol{y}_{j}, \boldsymbol{b}_{j}, \boldsymbol{c}_{j}} \sum_{t=1}^{T}\left[\log _{2}\left(1+\frac{P_{j}}{b_{j}(t)}\right)-\log _{2}\left(1+\frac{P_{j}}{c_{j}(t)}\right)\right] \\
& \text { s.t. } \quad \min _{\Delta x_{k}^{2}+\Delta y_{k}^{2} \leq \mathcal{R}_{k}^{2}} f\left(x_{j}(t), y_{j}(t)\right) \geq c_{j}(t), \forall t, k, \\
& \left(x_{j}(t)-x_{b}\right)^{2}+\left(y_{j}(t)-y_{b}\right)^{2}+H^{2}-b_{j}(t) \leq 0, \forall t, \\
& c_{j} \geq H^{2},(3), \forall t .
\end{aligned}
$$

Due to the infinite number of $\left(\Delta x_{k}, \Delta y_{k}\right)$ in (25b), we have to convert (25b) into equivalent constraints by substituting (1) and (2) into (25b)

$$
\begin{aligned}
& \Delta x_{k}^{2}+\Delta y_{k}^{2}-\mathcal{R}_{k}^{2} \leq 0, \forall k \\
& -\left(x_{j}(t)-x_{k}^{E}(t)-\Delta x_{k}\right)^{2}-\left(y_{j}(t)-y_{k}^{E}(t)-\Delta y_{k}\right)^{2} \\
& -H^{2}+c_{j}(t) \leq 0, \forall k
\end{aligned}
$$

Since there exists at least one point $\left(\Delta \dot{x}_{k}=0, \Delta \dot{y}_{k}=0\right)$ such that $\Delta \dot{x}_{k}^{2}+\Delta \dot{y}_{k}^{2}-\mathcal{R}_{k}^{2}<0$, we can utilize the S-procedure [31] to show that the implication (26a) $\Rightarrow(26 b)$ holds if and only if $\exists \lambda_{k}(t) \geq 0$ such that

$$
\mathbf{\Upsilon}\left(x_{j}(t), y_{j}(t), c_{j}(t), \lambda_{k}(t)\right) \succeq \mathbf{0}, \forall j, k
$$

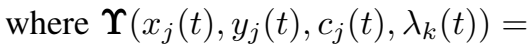

$$
\begin{aligned}
& {\left[\begin{array}{ccc}
\lambda_{k}(t)+1 & 0 & x_{k}^{E}(t)-x_{j}(t) \\
0 & \lambda_{k}(t)+1 & y_{k}^{E}(t)-y_{j}(t) \\
x_{k}^{E}(t)-x_{j}(t) & y_{k}^{E}(t)-y_{j}(t) & -\lambda_{k}(t) \mathcal{R}_{k}^{2}+\nu_{k}(t)
\end{array}\right] \text { and }} \\
& \nu_{k}(t)=\left(x_{j}(t)-x_{k}^{E}(t)\right)^{2}+\left(y_{j}(t)-y_{k}^{E}(t)\right)^{2}+H^{2}-c_{j}(t) .
\end{aligned}
$$

Then, we replace (25b) with (27) and introduce the slack variable $\boldsymbol{\Lambda}_{K \times T}=\left\{\lambda_{k}(t)\right\}_{k=1, t=1}^{K, T}$ into problem (25). However, the object function (25a) is non-concave since the first term is convex and the constraint (27) is non-convex since $\nu_{k}(t)$ is a non-linear function. Similar to [14], we assume a feasible point $\boldsymbol{x}_{j}^{f}=\left[x_{j}^{f}(1), \ldots, x_{j}^{f}(T)\right]^{\top}, \boldsymbol{y}_{j}^{f}=\left[y_{j}^{f}(1), . ., y_{j}^{f}(T)\right]^{\top}$, and $\boldsymbol{b}_{j}^{f}=\left[b_{j}^{f}(1), . ., b_{j}^{f}(T)\right]^{\top}$ for (25). The first-order Taylor expansions of the first term of (25), $x_{j}^{2}(t), y_{j}^{2}(t)$ at the above feasible point can be written as

$$
\log _{2}\left(1+\frac{P_{j}}{b_{j}(t)}\right) \geq \log _{2}\left(1+\frac{P_{j}}{b_{j}^{f}(t)}\right)-\frac{P_{j}\left(b_{j}(t)-b_{j}^{f}(t)\right)}{\ln 2\left(P_{j}+b_{j}^{f}(t)\right) b_{j}^{f}(t)},
$$

$x_{j}^{2}(t) \geq x_{j}^{f}\left(2 x_{j}(t)-x_{j}^{f}\right), y_{j}(t) \geq y_{j}^{f}\left(2 y_{j}(t)-y_{j}^{f}\right)$.
In this way, we approximately convert the problem (25) into

$$
\begin{array}{ll}
\max _{\boldsymbol{x}_{j}, \boldsymbol{y}_{j}, \boldsymbol{b}_{j}, \boldsymbol{c}_{j}, \boldsymbol{\Lambda}} \sum_{t=1}^{T} \frac{-P_{j}\left(b_{j}(t)-b_{j}^{f}(t)\right)}{\ln 2\left(P_{j}+b_{j}^{f}(t)\right) b_{j}^{f}(t)}-\log _{2}\left(1+\frac{P_{j}}{c_{j}(t)}\right) \\
\text { s.t. } \quad \tilde{\boldsymbol{\Upsilon}}\left(x_{j}(t), y_{j}(t), c_{j}(t), \lambda_{k}(t)\right) \succeq \mathbf{0}, \forall j, k \\
\quad \lambda_{k}(t) \geq 0,(25 c),(25 d) .
\end{array}
$$

where the $\tilde{\Upsilon}$ can be obtained by substituting $\widetilde{\nu}_{k}(t)=$ $x_{j}^{f}\left(2 x_{j}(t)-x_{j}^{f}\right)-x_{k}^{E}(t)\left(x_{k}^{E}(t)-2 x_{j}(t)\right)+y_{j}^{f}\left(2 y_{j}(t)-y_{j}^{f}\right)-$ $y_{k}^{E}(t)\left(y_{k}^{E}(t)-2 y_{j}(t)\right)+H^{2}-c_{j}(t)$ with $\nu_{k}(t)$ in $\Upsilon$. Note that $\widetilde{\Upsilon} \succeq \Upsilon$. i.e., (31b) implies (27), since (30) is satisfied. We observe that problem (31) is a semidefinite programming problem whose optimal solution can be obtained by interiorpoint method [31]. Thus, we can approximately obtain a suboptimal solution to (25a).

\section{Popular Matching based Channel Allocation}

In this section, we simultaneously optimize the channel allocation variable $\boldsymbol{\omega}$ for both (13) and (14) under given trajectory and power. Note that (13) and (14) are jointly optimized, i.e., each UAV aims at optimizing its secrecy rate while each D2D user aims at optimizing its achievable rate, which can be viewed as a multi-objective optimization problem. Matching theory can be utilized to optimize the individual utilities for each side of agents [18]. In matching theory, both sides of agents are rational and focus on their own benefits. From the perspective of UAVs, they want to recruit appopriate D2D users as friendly jammers to benefit themselves. However, the utilities of UAVs cannot be unilaterally improved, since their interests conflict with that of D2D users. Due to the cochannel interference, unilaterally optimizing (13) may degrade the achieved performance of (14), and vice versa. Hence, the obtained stable solution achieved by matching theory is the Pareto optimal, i.e., no one can unilaterally improve their utilities without reducing the utilities of others, which can strike the trade-off between (13) and (14). Compared to unilaterally optimizing the utilities of one side of users, the stable solution is more suitable for the distributed scenario.

In the following subsections, we first introduce the popular matching under the background of SIoFT, and then propose a hierarchical matching algorithm to initially construct the maximum popular matching structure without considering the externalities. Finally, we will introduce how to maintain the popularity of one matching against externalities.

\section{A. Preliminaries}

It is obvious that the problem (13) and (14) can be formulated as the classic stable roommates problem, where many tenants can live in one house, and one house can be occupied by many tenants within its quota [23]. As such, UAVs and D2D users can be abstracted as landlords and tenants, respectively. This kind of matching problem can be categorized as a two-sided matching between two disjoint sets of agents where the agents of one side rank the agents of the opposite side based on their preferences. Herein, the matching between UAVs and D2D users is to maximize its own utility. The utility function $U_{i}\left(u_{j}\right)$ 
for $d_{i}$ is denoted by its date rate when reusing $u_{j}$ 's spectrum, i.e., $U_{i}\left(u_{j}\right)=\log _{2}\left(1+\frac{P_{i} g_{d_{i}^{T}, d_{i}^{R}}(t)}{I_{j, i}(t)+N_{0}}\right)$. And the utility function $U_{j}\left(d_{i}\right)$ for $u_{j}$ is measured by the real-time secrecy rate. i.e., $U_{j}\left(d_{i}\right)=\log _{2}\left(1+\frac{P_{j}(t) g_{j, b}(t)}{P_{i} g_{i, j}(t)+N_{0}}\right)-$

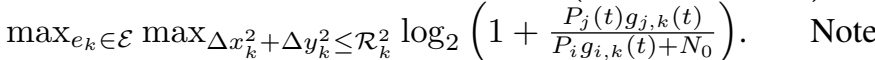
that the UAV only considers the independent influence when one D2D user requests to reuse its spectrum, so does this D2D user. This setting can guarantee that the preference lists are fixed and the optimization problem of matching keeps the similar as problems (13) and (14), except that the co-channel interferences of other D2D users are simplified. We further use the symbol $\succ$ to represent the preference relation. For example, $\succ_{i}$ denotes the preference relations of $d_{i} \in \mathcal{D}$, and $j \succ_{i} j^{\prime}$ denotes that $d_{i}$ prefers reusing the spectrum resource of $u_{j}$ to $u_{j^{\prime}}$, i.e., $U_{i}\left(u_{j}\right)>U_{i}\left(u_{j^{\prime}}\right)$. Similarly, $i \succ_{j} i^{\prime}$ denotes that $u_{j}$ prefers sharing the spectrum resource with $d_{i}$ to $d_{i^{\prime}}$. Herein, since UAVs will share spectrum resource with a subset of D2D users $\Phi_{j}(\mathcal{D}) \in \mathcal{D}, \Phi_{j}(\mathcal{D}) \succ_{j} \Phi_{j}\left(\mathcal{D}^{\prime}\right)$ denotes that $u_{j}$ prefers sharing the spectrum resource with the subset $\Phi_{j}(\mathcal{D})$ to $\Phi_{j}\left(\mathcal{D}^{\prime}\right)$. Based on the above discussion, the two-sided many-to-one matching is defined as

Definition 1: The two-sided many-to-one matching $\Omega$ is defined as a function mapping from $\mathcal{D} \cup \mathcal{U} \cup \varnothing$ into $\mathcal{D} \cup \mathcal{U} \cup \varnothing$ such that for $d_{i} \in \mathcal{D}$ and $\left.u_{j} \in \mathcal{U}: 1\right)\left|\Omega\left(d_{i}\right)\right| \leq 1, \forall d_{i} \in \mathcal{D}$ and $\Omega\left(d_{i}\right)=\emptyset$, if $\left.\Omega\left(d_{i}\right) \notin \mathcal{D} ; 2\right)\left|\Omega\left(u_{j}\right)\right| \leq q_{j}, \forall u_{j} \in \mathcal{U}$, and $\Omega\left(u_{j}\right)=\emptyset$, if $\left.\Omega\left(u_{j}\right) \notin \mathcal{D} ; 3\right) \Omega\left(d_{i}\right)=u_{j}$, only if $u_{j} \in \Omega\left(u_{i}\right)$, i,e., $\omega_{i, j, t}=1$, where $|\cdot|$ refers to the cardinality of a set.

We can note that (13c) and (14c) are satisfied through the three conditions in Definition 1. Besides, the matching is feasible only when the constraints (13b), (13d), and (14b) are met. To this end, we delete those agents who cannot satisfy constraints (13b), (13d), and (14b) in each agent's preference list, and thus no one will violate the constraints.

Definition 2: Given a matching $\Omega$, if an unmatched pair $\left(d_{i}, u_{j}\right)$ satisfies both $j \succ_{i} \Omega\left(d_{i}\right)$ and $i \succ_{j} k, \exists d_{k} \in \Omega\left(u_{j}\right)$ or $\left|\Omega\left(u_{j}\right)\right|<q_{j}$, the pair $\left(d_{i}, u_{j}\right)$ composes a blocking pair in $\Omega$. A stable matching does not involve any blocking pair.

Definition 2 refers to the stability condition of a matching, which also indicates the weak Pareto optimality, since no one can be better of by deviating to another choice under stability condition [23]. Although the stable matching can ensure the weak Pareto optimality, it does not ensure that UAVs will reach their capacity, i.e., quota. Intuitively, the secrecy rate of one UAV may be further improved by sharing spectrum with more friendly jammers which can launch powerful attack to eavesdroppers while interfering weakly with this UAV. Hence, stability is not desirable by both UAVs and D2D users. On one hand, the UAVs would like to share their spectrum with more D2D users and thus the security performance may be improved by the addition of more friendly jammers. On the other hand, more D2D users can meet their achievable rate requirements by joining in the spectrum sharing. For this purpose, we relax the stability condition (local stability) to the popularity condition (global stability) [25].

\section{B. Popular Matching without Externalities}

Given two matchings $\Omega_{0}$ and $\Omega_{1}$, we say that one agent $u$ prefers $\Omega_{0}$ to $\Omega_{1}$ if $u$ benefits more in $\Omega_{0}$ than $\Omega_{1}$. Let $\varphi\left(\Omega_{0}, \Omega_{1}\right)$ denote the number of agents preferring $\Omega_{0}$ to $\Omega_{1}$. Hence, $\Omega_{0}$ is more popular than $\Omega_{1}$ if $\varphi\left(\Omega_{0}, \Omega_{1}\right)>\varphi\left(\Omega_{1}, \Omega_{0}\right)$

Definition 3 (Majority Rule): The matching $\Omega$ is popular if $\varphi\left(\Omega, \Omega^{\prime}\right) \geq \varphi\left(\Omega^{\prime}, \Omega\right)$ for any matching $\Omega^{\prime}$ derived from $\mathcal{D} \cup \mathcal{U} \cup \emptyset$.

In other words, a popular matching $\Omega$ satisfies that no matching is preferred by more agents than in $\Omega$. In [24], the authors demonstrated that the stability condition is very strong, and the stable matching is the minimal size popular matching. For ease of understanding this statement, there exist two disjoint sets $\mathcal{D}=\left\{d_{1}, d_{2}\right\}$ and $\mathcal{U}=\left\{u_{1}, u_{2}\right\}$, the preference relation for $d_{1}$ is $u_{2} \succ_{d_{1}} u_{1} \succ_{d_{1}} \emptyset$, for $d_{2}$ is $u_{1} \succ_{d_{2}} \emptyset$ (i.e., $d_{2}$ prefers $u_{1}$ to being unmatched, and $u_{2}$ is not in the preference since these constraints will be violated), for $u_{1}$ is $d_{1} \succ_{u_{1}} \emptyset$, and for $u_{2}$ is $d_{1} \succ_{u_{2}} d_{2}$. We can derive a stable matching $\Omega_{s}=\left\{\left(d_{1}, u_{2}\right)\right\}$ and a maximum size popular matching $\Omega_{p}=\left\{\left(d_{1}, u_{1}\right),\left(d_{2}, u_{2}\right)\right\}$. Though $\varphi\left(\Omega_{s}, \Omega_{p}\right)=\varphi\left(\Omega_{p}, \Omega_{s}\right)$, $\Omega_{s}$ may achieve the better performance.

In order to facilitate the analysis, we introduce the vote function for UAVs and D2D users to vote for preferred matching. For a D2D user $d_{i}$, the vote function $v_{i}\left(\Omega\left(d_{i}\right), \Omega^{\prime}\left(d_{i}\right)\right)=1$ if $\Omega\left(d_{i}\right) \succ_{d_{i}} \Omega^{\prime}\left(d_{i}\right), v_{i}\left(\Omega\left(d_{i}\right), \Omega^{\prime}\left(d_{i}\right)\right)=-1$ if $\Omega^{\prime}\left(d_{i}\right) \succ_{d_{i}}$ $\Omega\left(d_{i}\right)$, and $v_{i}\left(\Omega\left(d_{i}\right), \Omega^{\prime}\left(d_{i}\right)\right)=0$ if $d_{i}$ is indifferent between two matchings. Similarly, the vote function of UAV $u_{j}$ is expressed as $v_{j}\left(\Omega\left(u_{j}\right), \Omega^{\prime}\left(u_{j}\right)\right)$, calculated in the same way. As such, the popularity deviation can be defined as

$$
\varphi\left(\Omega, \Omega^{\prime}\right)=\sum_{d_{i} \in \mathcal{D}} v_{i}\left(\Omega\left(d_{i}\right), \Omega^{\prime}\left(d_{i}\right)\right)+\sum_{u_{j} \in \mathcal{U}} v_{j}\left(\Omega\left(u_{j}\right), \Omega^{\prime}\left(u_{j}\right)\right) .
$$

Note that the set of all devices and their preference relations can be abstracted as a graph $\mathcal{G}=\{(\mathcal{D} \cup \mathcal{U}, \mathcal{E})\}$, where $\mathcal{D} \cup \mathcal{U}$ denotes the vertex set and $\mathcal{E}$ denotes the edge set. Next, we propose a distributed algorithm to search for a maximum size popular matching. In [26], the authors proposed an algorithm that computes a maximum one-to-one popular matching with linear time complexity. Based on [26], we aim to convert the many-to-one matching to one-to-one matching, by assuming that each $u_{j}$ has $q_{j}$ copies and each copy represents a virtual UAV which can occupy only one D2D user, i.e., $u_{j}=\left\{u_{j}^{1}, \ldots, u_{j}^{q_{j}}\right\}$. Based on [26], we further set that each $d_{i}$ has two copies, i.e., $d_{i}=\left\{d_{i}^{1}, d_{i}^{2}\right\}$. In this way, we convert the original $\mathcal{G}$ to $\mathcal{G}^{\prime}=\left\{\left(\mathcal{D}^{\prime} \cup \mathcal{U}^{\prime}, \mathcal{E}^{\prime}\right)\right\}$, where $\mathcal{D}^{\prime}$ consists of a set of D2D pairs that contain two copies, and a set of UAVs that contain $q_{j}$ copies. The edge set $\mathcal{E}^{\prime}$ is defined as $\mathcal{E}^{\prime}=\left\{\left(u_{j}^{f}, d_{i}^{l}\right):\left(u_{j}, d_{i}\right) \in \mathcal{E}, f \in\left\{1, \ldots, q_{j}\right\}, l \in\{1,2\}\right\}$. Based on the utility function values, the preference relations of each $d_{i}^{l}$ for two different UAVs remain unchanged, i.e., $u_{j}^{f} \succ_{d_{i}^{l}} u_{j^{\prime}}^{h}$ if $u_{j} \succ_{d_{i}} u_{j^{\prime}}$, for any $f \in\left\{1, \ldots, q_{j}\right\}$ and $h \in\left\{1, \ldots, q_{j^{\prime}}\right\}$. However, the utility values of $d_{i}$ reusing any two copies of $u_{j}$ are equal but $d_{i}$ can only select one copy of $u_{j}$. Hence, the preference relations of each $d_{i}^{l}$ for the same UAV are based on the ranking of id numbers in a descending order, i.e., $u_{j}^{1} \succ_{d_{i}^{l}} u_{j}^{2} \succ, \ldots, \succ u_{j}^{q_{j}}$. For the UAV 
$u_{j}^{f}$, its preference relations for two different D2D users with the same id numbers remain unchanged, i.e., $d_{i}^{l} \succ_{u_{j}^{f}} d_{i^{\prime}}^{l}$ if $d_{i} \succ_{u_{j}} d_{i^{\prime}}$, for $l \in\{1,2\}$. The preference relations for the same D2D user or two different D2D users with the different id numbers are based on the ranking of id numbers in an ascending order, i.e., $d_{i}^{2} \succ_{u_{j}^{f}} d_{k}^{1}$, for any $i=k$ or $i \neq k$.

Herein, we propose a hierarchical matching (HM) algorithm as shown in Alg.1. The superscript $l$ in $d_{i}^{l}$ denotes the layer number, i.e., $l=1$ refers to the bottom layer while $l=2$ refers to the top layer. Initially, all layer-1 D2D users become active and meanwhile all layer-2 users stay inactive. The aim of this hierarchy is to promote the D2D users rejected by all UAVs through deleting these D2D users from layer-1 and only reserving the layer-2 version of these D2D users, which are more preferred by the UAVs than the layer-1 version. Besides, we use a queue $Q$ containing both the set of active and unmatched D2D users. The main idea of this hierarchical matching algorithm is summarized as

- Initially, only those D2D users in layer-1 are active and then request spectrum sharing to their most preferred UAVs to which they have not requested yet.

- Whenever an active D2D user $d_{i}^{1}$ in layer-1 has been rejected by all UAVs, $d_{i}^{1}$ is removed from $Q$ and $d_{i}^{2}$ is pushed into $Q$.

- Whenever a UAV $u_{j}^{f}$ receives a spectrum sharing request from a D2D user, $u_{j}^{f}$ accepts or rejects this request based on its preference relations.

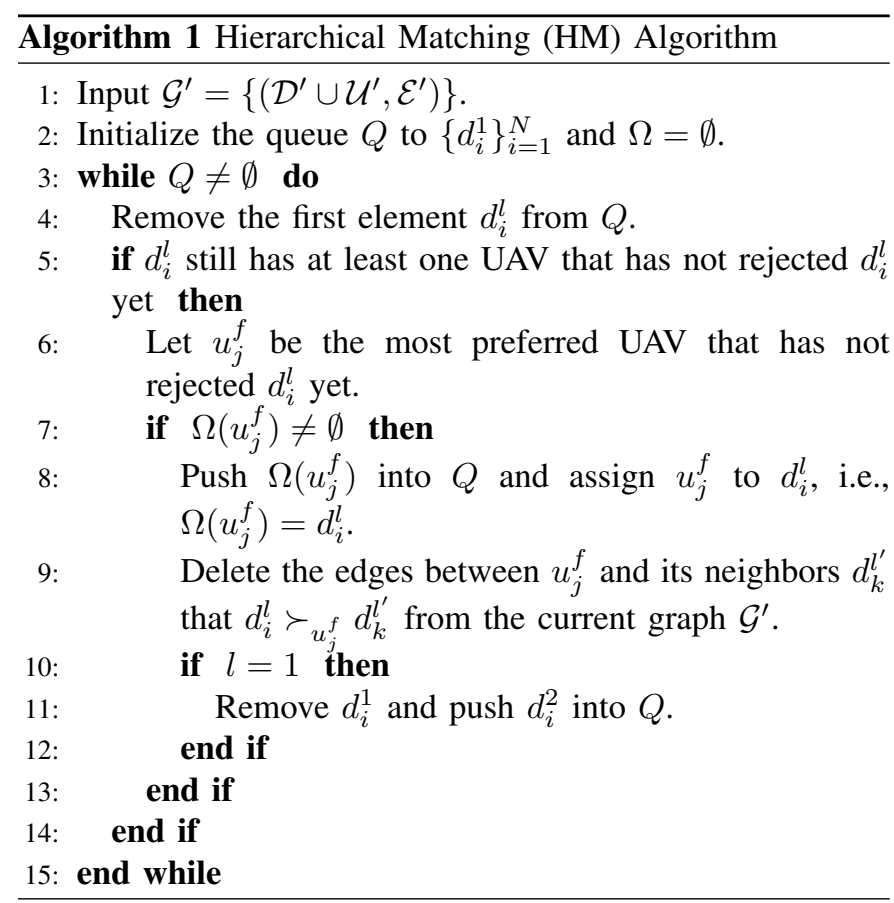

Computational Complexity of Alg. 1: The complexity for building the graph $\mathcal{G}^{\prime}$ is $\mathcal{O}\left(\left|\mathcal{E}^{\prime}\right|+\left|\mathcal{D}^{\prime} \cup \mathcal{U}^{\prime}\right|\right)$, for matching is $\mathcal{O}\left(\left|\mathcal{D}^{\prime}\right| \cdot\left|\mathcal{U}^{\prime}\right|\right)$. So the complexity taken to compute $\Omega$ is $\mathcal{O}\left(\max \left\{\left|\mathcal{E}^{\prime}\right|+\left|\mathcal{D}^{\prime} \cup \mathcal{U}^{\prime}\right|,\left|\mathcal{D}^{\prime}\right| \cdot\left|\mathcal{U}^{\prime}\right|\right\}\right)$.

Next, we prove that the computed matching $\Omega$ is a maximum size popular matching with respect to original graph $\mathcal{G}$. Firstly, we partition $D$ into the set $D_{1}$ of the layer-1 D2D users and the set $D_{2}$ of the layer-2 D2D users. Similarly, those UAVs matched to the layer-1 and layer-2 D2D users are in the set $U_{1}$ and $U_{2}$, respectively. Note that both the conditions $D_{1} \cup D_{2}=$ $\emptyset$ and $U_{1} \cup U_{2}=\emptyset$ are satisfied.

Claim 1: All the unmatched D2D users in $\Omega$ are in $D_{2}$ while all the unmatched UAVs are in $U_{1}$.

Label each edge $e=(d, u)$ in $\mathcal{E}$ by $\left(\sigma_{e}, \beta_{e}\right)$, where $\sigma_{e}=v_{d}(u, \Omega(d))$ and $\beta_{e}=v_{u}(d, \Omega(u))$ are the vote functions defined before. Note that an $e$ is a blocking edge only if $\left(\sigma_{e}, \beta_{e}\right)$ is labeled $(1,1)$, since both $d$ and $u$ prefer each other to their current partners.

Lemma 2: $\forall e \in D_{2} \times U_{1},\left(\sigma_{e}, \beta_{e}\right)$ is labeled $(-1,-1)$. $\forall e \in D_{1} \times U_{2},\left(\sigma_{e}, \beta_{e}\right)$ is labeled $(1,1)$.

Proof: To prove the first condition, we let $e=(d, u) \in$ $D_{2} \times U_{1} . d$ must be matched in $\Omega$, and otherwise $d^{2}$ would have requested to $u$ when $d^{1}$ was active. However, the condition $u \in U_{1}$ indicates that $u$ has not received the request from any layer- 2 user, and otherwise $u$ will belong to $U_{2}$. Thus, $d^{2}$ must be matched to a UAV $u^{\prime}$ that is preferred to $u$ for $d^{2}$ so that $v_{d}(u, \Omega(d))=-1$. Similarly, considering that $d^{1}$ must be rejected by all UAVs including $u, u$ must be matched to a user that is preferred to $d^{1}$ for $u$. Since $u$ has not received the request from any layer-2 user, $u$ prefers $\Omega(u)$ to $d$ so that $v_{u}(d, \Omega(u))=-1$.

For the second condition, we can see that no UAV in $U_{1}$ receives any request from a layer- 2 user, and thus $\Omega$ restricted to the set $D_{1} \times U_{1}$ is popular since those UAVs in $U_{1}$ only receive the requests from layer- 1 users and accept the most preferred one. Thus, the matching restricted to $D_{1} \times U_{1}$ contains no blocking edges. Besides, the D2D users in $D_{2}$ request in line with their preferences while the UAVs prefer layer-2 users to layer-1 users, which indicates that $D_{2} \times U_{2}$ also contains no blocking edges labeled $(1,1)$. By elimination rule, if there exists an edge labeled $(1,1)$, it must belong to $D_{1} \times U_{2}$.

Let $\mathcal{G}_{\Omega}$ represent the subgraph of $\mathcal{G}$ by removing all edges labeled $(-1,-1)$ from $\mathcal{G}$. Based on the definitions in graph theory [32], a path or circle where alternate edges belong to $\Omega$ is called an alternating path or circle in $\Omega$. If the endpoints of this alternating path are unmatched in $\Omega$, this path is called augmenting path. Just as its name suggests, an alternating path starts with an unmatched node, and its edges belong alternately to $\Omega$ and not to $\Omega$.

Lemma 3: Given an alternating path $\rho=$ $\left\langle y_{0}, x_{1}, y_{1}, x_{2}, y_{2}, \ldots,\right\rangle$ with respect to $\mathcal{G}_{\Omega}$, where $\left(x_{i}, y_{i}\right)$ is matched in $\Omega$, for $i>0$, the following conditions must be satisfied.

1) If $y_{0} \in D_{2} \cup U_{1}$, no edge in $\rho$ is labeled $(1,1)$;

2) If $y_{0} \in D_{1} \cup U_{2}$, at most one edge in $\rho$ is labeled $(1,1)$.

Proof: For the first condition, if $y_{0} \in D_{2}$, it is easy to derive by Lemma 2 that no edges in $\mathcal{G}_{\Omega}$ belong to $D_{2} \times U_{1}$. Hence, the $y_{0}$ 's neighbor $x_{1}$ in $\rho$ belongs to $U_{2}$. Since $\left(x_{i}, y_{i}\right)$ is matched in $\Omega$, all the matched partner must belong to $D_{2} \times U_{2}$ for $i>0$. According to Lemma 2 , the edges labeled $(1,1)$ must belong to $D_{1} \times U_{2}$, and thus no edge in $\rho$ is labeled $(1,1)$. If $y_{0} \in U_{1}$, no edges belong to $D_{2} \times U_{1}$ since all edges labeled $(-1,-1)$ are removed from $\mathcal{G}$. Thus, all the matched partner 
must belong to $D_{1} \times U_{1}$ for $i>0$, and similarly no edge in $\rho$ is labeled $(1,1)$.

For the second condition, if $y_{0} \in D_{1}$, no edges in $\mathcal{G}_{\Omega}$ belong to $D_{1} \times U_{2}$. Once an edge in $D_{1} \times U_{2}$ is traversed, $\rho$ gets stuck in $D_{2} \times U_{2}$ as the earlier case. Thus, only one edge $\left(y_{0}, x_{1}\right)$ can be labeled $(1,1)$ when $x_{1} \in U_{2}$. If $y_{0} \in D_{2}$, only one edge $\left(y_{0}, x_{1}\right)$ can be labeled $(1,1)$ when $x_{1} \in D_{1}$.

For the ease of analysis, we define two types of alternating paths. The type- 1 alternating path satisfies $y_{0} \in D_{2} \cup U_{1}$ and type-2 satisfies $y_{0} \in D_{1} \cup U_{2}$. Given any matching $\Omega^{\prime} \in \mathcal{G}$, we can restrict it to $\mathcal{G}_{\Omega}$ by deleting the edges labeled $(-1,-1)$ and assuming that the agents involving these deleted edges are unmatched in $\Omega^{\prime}$ (i.e., this modification to the calculation of the popularity deviation $\varphi\left(\Omega, \Omega^{\prime}\right)$. Hence, $\Omega \oplus \Omega^{\prime}$ is also in $\mathcal{G}_{\Omega}$, which represents the collection of alternating paths. Now, we prove that $\varphi\left(\Omega^{\prime}, \Omega\right) \leq \varphi\left(\Omega, \Omega^{\prime}\right)$ through Theorem 1 .

Theorem 1: $\forall \Omega^{\prime} \in \mathcal{G}_{\Omega}$, the following conditions are always satisfied:

1) If $\rho$ is an alternating cycle in $\Omega \oplus \Omega^{\prime}, \varphi(\Omega \oplus \rho, \Omega) \leq$ $\varphi(\Omega, \Omega \oplus \rho)$;

2) If $\rho$ is an alternating path in $\Omega \oplus \Omega^{\prime}$, there exists at least one endpoint of $\rho$ is unmatched in $\Omega$, and $\varphi(\Omega \oplus \rho, \Omega) \leq$ $\varphi(\Omega, \Omega \oplus \rho)$;

3) If $\rho$ is an alternating path in $\Omega \oplus \Omega^{\prime}$, both endpoints of $\rho$ are matched in $\Omega$, and $\varphi(\Omega \oplus \rho, \Omega) \leq \varphi(\Omega, \Omega \oplus \rho)$.

Proof: Since $\varphi(\Omega \oplus \rho, \Omega)-\varphi(\Omega, \Omega \oplus \rho)=$ $\sum_{u \in \rho} v_{u}\left(\Omega^{\prime}(u), \Omega(u)\right)$, the clear expression can be given by

$$
\sum_{u \in \rho} v_{u}\left(\Omega^{\prime}(u), \Omega(u)\right)=\sum_{u^{\prime} \in \rho}(-1)+\sum_{e \in \rho \cap \Omega^{\prime}}\left(\sigma_{e}+\beta_{e}\right),
$$

where $u^{\prime}$ is unmatched in $\Omega^{\prime}$. For $e=(x, y), \sigma_{e}=v_{x}(y, \Omega(x))$ and $\beta_{e}=v_{y}(x, \Omega(y))$.

For the first condition, since every edge in $\Omega$ is either in $D_{1} \times U_{1}$ or $D_{2} \times U_{2}$, the alternating cycle $\rho$ must contain a vertex $x \in D_{2} \times U_{1}$, and thus $\rho \backslash(x, \Omega(x))$ is a type-1 alternating path. Hence, no edge in $\rho \backslash(x, \Omega(x))$ is labeled $(1,1)$ so that $\sigma_{e}+\beta_{e} \leq 0$. Thus, $\varphi(\Omega \oplus \rho, \Omega) \leq \varphi(\Omega, \Omega \oplus \rho)$.

For the second condition, based on Claim 1, the unmatched endpoint must belong to $D_{2} \times U_{1}$ and thus $\rho$ is a type-1 path. Thus, $\varphi(\Omega \oplus \rho, \Omega) \leq \varphi(\Omega, \Omega \oplus \rho)$.

For the third condition, since both endpoints of $\rho$ are matched in $\Omega$, neither endpoint is matched in $\Omega^{\prime}$ and contribute -1 to the first term on (33). Through Lemma 3, at most one edge can be labeled $(1,1)$, and then this contribution is canceled out by the votes of two endpoints. The condition $\varphi(\Omega \oplus \rho, \Omega) \leq \varphi(\Omega, \Omega \oplus \rho)$ still holds.

Based on Theorem 1, assuming that $\Omega^{\prime} \cap \mathcal{E}_{\Omega}$ denotes the intersection of $\Omega^{\prime}$ and the edge set of $\mathcal{G}_{\Omega}$, we have

$$
\begin{aligned}
\varphi\left(\Omega, \Omega^{\prime}\right) & =\sum_{\rho \in \Omega \oplus\left(\Omega^{\prime} \cap \mathcal{E}_{\Omega}\right)} \varphi(\Omega, \Omega \oplus \rho) \\
& \geq \sum_{\rho \in \Omega \oplus\left(\Omega^{\prime} \cap \mathcal{E}_{\Omega}\right)} \varphi(\Omega \oplus \rho, \Omega)=\varphi\left(\Omega^{\prime}, \Omega\right) .
\end{aligned}
$$

Therefore, the popularity of $\Omega$ computed by Alg. 1 is proved. Next, we will prove that $\Omega$ is the maximum size popular matching.

Lemma 4: There exists no augmenting path for $\Omega$ with respect to $\mathcal{G}_{\Omega}$.
Proof: Based on Claim 1, if there is an augmenting path $p$ for $\Omega$, for two neighbor vertices $y_{i}$ and $x_{i+1}$, one belongs to $D_{2}$ and the other belongs to $U_{1}$, since there only exist edges belonging to $\left(D_{1} \times U_{1}\right) \cup\left(D_{2} \times U_{2}\right)$ in $\Omega$. But the edge in $\left(D_{2} \times U_{1}\right)$ is removed from $\Omega$, which contradicts the condition.

Lemma 5: Given a matching $\Omega^{\prime}$ in $\mathcal{G}, \varphi\left(\Omega, \Omega^{\prime}\right)>0$ if $\left|\Omega^{\prime}\right|>|\Omega|$.

Proof: Suppose that $\Omega^{\prime}$ satisfies the condition $\left|\Omega^{\prime}\right| \geq|\Omega|$. Based on the deductions in graph theory [32], there must exist an augmenting path $p \in \Omega \oplus \Omega^{\prime}$ in $\mathcal{G}$. By restricting $\Omega^{\prime}$ to $\mathcal{G}_{\Omega}$, there exists no augmenting path for $\Omega$ with respect to $\mathcal{G}_{\Omega}$, and $p$ is partioned into sub-paths $p_{1}, p_{2}, \ldots, p_{s}$ in $\mathcal{G}_{\Omega}$ in which both $p_{1}$ and $p_{s}$ have one unmatched endpoint in $\Omega$. Through Claim 1 and Lemma 2, the endpoint belongs to $\left(D_{2} \times U_{1}\right)$, and there is no edge labeled $(1,1)$ in these two sub-paths. For $p_{1}$ and $p_{s}$, all edges except for one endpoint $x$ are labeled $(1,-1)$. Since $x$ is matched in $\Omega, \varphi\left(\Omega, \Omega \oplus p_{1}\right)=1+\varphi\left(\Omega \oplus p_{1}, \Omega\right)$.

For another alternating path $p_{k}$ and $k=\{2, \ldots, s-1\}$, we can prove that $\varphi\left(\Omega, \Omega \oplus p_{k}\right)>\varphi\left(\Omega \oplus p_{k}, \Omega\right)$ by Theorem 1 . And thus $\Omega$ is the maximum size popular matching.

\section{Popular Matching with Externalities}

Considering the co-channel interference, the preference of each user is dynamically changing, which causes the externalities in matching theory [18]. The externalities indicate that the utilities of resource users (D2D users) are influenced not only by resource owners' decisions, but also by those resource users reusing the same resource. The HM algorithm can initially construct the maximum popular matching structure as far as possible. In this section, we will investigate how to maintain the popularity upon the initial matching $\Omega$ obtained by HM algorithm. We conceive the vote deviation with the framework of directed graph in graph theory. Different from the fixed preferences defined before, the utility functions of UAV $U_{j}(t)$ can be denoted as $U_{j}(t)=\left[R_{j, b}(t)-\max _{e_{k} \in \mathcal{E}} \max _{\Delta x_{k}^{2}+\Delta y_{k}^{2} \leq \mathcal{R}_{k}^{2}} R_{j, k}(t)\right]^{+}$with constraints (13b)-(13d) ensured, while the utility functions of D2D user $U_{i}(t)=R_{i}(t)$ can be denoted as with constraints (14b) and (14c) ensured.

Definition 4: Given a set of users $\left\{d_{1}, d_{2}, \ldots, d_{i}\right\}$ in different spectrum sharing groups (i.e., each UAV represents a group), if we change the spectrum sharing strategy from $\pi$ to $\hat{\pi}$ by $\left(d_{1} \rightarrow\right.$ $\left.\pi_{d_{2}}\right),\left(d_{2} \rightarrow \pi_{d_{3}}\right), \ldots,\left(d_{i-1} \rightarrow \pi_{d_{i}}\right),\left(d_{i} \rightarrow \pi_{d_{1}}\right)$, and vote deviation $\varphi\left(\Omega^{\pi \rightarrow \hat{\pi}}, \Omega\right)>\varphi\left(\Omega, \Omega^{\pi \rightarrow \pi}\right)$, these $i$ users compose a rotation-swap coalition.

Here, we use $\left(d_{1} \rightarrow \pi_{d_{2}}\right)$ to denote the operation that $d_{1}$ leaves the spectrum sharing group $\pi_{d_{1}}$ involving $d_{1}$ and joins the spectrum sharing group $\pi_{d_{2}}$ involving $d_{2}$.

To describe the situation that one user $d_{1}$ joins in another group $\pi_{d_{i}}$ without replacing any user included in $\pi_{d_{i}}$ when the quota of $\pi_{d_{i}}$ is still not fulfilled, i.e., $\left(d_{1} \rightarrow \pi_{d_{i}}\right),\left(\emptyset \rightarrow \pi_{d_{1}}\right)$, we create a virtual user for those groups whose quotas are still not fulfilled, and let $\mathcal{D}_{v}=\left\{d_{v}\right\}_{v=1}^{m}$ for $m \leq M$ denote the virtual user set. Let $\widetilde{\mathcal{D}}=\left(\mathcal{D}_{v} \cup \mathcal{D}\right)$ denote all users involved. Let the value function of each virtual user $d_{v}$ for any two matchings equal to 0 , i.e., the preferences of active users are not affected by virtual users. 
Definition 5: A matching $\Omega^{\pi}$ is a popular matching with externalities if there exists no rotation-swap coalition after executing spectrum sharing strategy $\boldsymbol{\pi}$.

We further use $d_{i} \rightarrow d_{k}$ to represent the operation that $d_{i}$ joins in $\pi_{d_{k}}$ and $d_{k}$ leaves $\pi_{d_{k}}$, where $\pi_{d_{i}} \in \Omega\left(d_{i}\right)$ and $\pi_{d_{k}} \in \Omega\left(d_{k}\right)$. The difference vote function of $d_{i}$ before and after $d_{i} \rightarrow d_{k}$ can be derived as

$$
\begin{aligned}
& \Delta_{d_{i}}\left(d_{i} \rightarrow d_{k}, \boldsymbol{\pi}_{-\left\{d_{i}, d_{k}\right\}}\right) \\
& =\varphi\left(\pi_{d_{i}} \in \Omega\left(d_{k}\right), \pi_{d_{k}} \notin \Omega\left(d_{k}\right), \boldsymbol{\pi}_{-\left\{d_{i}, d_{k}\right\}}\right) \\
& -\varphi\left(\pi_{d_{i}} \in \Omega\left(d_{i}\right), \boldsymbol{\pi}_{-\left\{d_{i}\right\}}\right) \\
& =\sum_{d_{x} \in \Omega\left(d_{i}\right) \cup \Omega\left(d_{k}\right)} v_{x}\left(\Omega^{d_{i} \rightarrow d_{k}}, \Omega\right) \\
& +\sum_{u_{j} \in\left\{\Omega\left(d_{i}\right), \Omega\left(d_{k}\right)\right\}} v_{u_{j}}\left(\Omega^{d_{i} \rightarrow d_{k}}, \Omega\right)
\end{aligned}
$$

In [33], the authors use a directed graph to model the externalities in stable matching model. Inspired by this idea, we construct a directed graph $\mathcal{G}^{D}\left(\mathcal{V}^{D}, \mathcal{E}^{D}\right)$ to model the externalities in our popular matching model, where $\mathcal{V}^{D}=\widetilde{\mathcal{D}}$ denotes the vertex set and $\mathcal{E}$ denotes the directed edge set. The directed edge exists between two users in different spectrum sharing group while the direction is from $d_{i}$ to $d_{j}$ if $\Delta_{d_{i}}\left(d_{i} \rightarrow d_{k}, \boldsymbol{\pi}_{-\left\{d_{i}, d_{k}\right\}}\right)>0$. Let edge weight $w_{i, j}=\Delta_{d_{i}}\left(d_{i} \rightarrow d_{k}, \boldsymbol{\pi}_{-\left\{d_{i}, d_{k}\right\}}\right)$.

Lemma 6: Given $i$ users in different groups, the rotationswap coalition can be abstracted as a positive directed loop in $\mathcal{G}^{D}$.

Proof: It is easy to prove that the vote deviation of a rotation-swap coalition $\varphi\left(\Omega^{\pi \rightarrow \hat{\pi}}, \Omega\right)=\sum_{k=1}^{i} w_{k^{\prime}, k}$, where $k^{\prime}=((k-2) \bmod i)+1$. Thus, the proof of Lemma 6 is concluded.

Corollary 1: If there exists a positive directed loop $d_{1} \rightarrow$ $d_{2}, \ldots, d_{i} \rightarrow d_{1}$ in $\mathcal{G}^{D}$, the popularity of this matching $\Omega$ can be improved by $\Omega^{\pi \rightarrow \hat{\pi}}=\left\{\left(d_{1}, \Omega\left(d_{2}\right)\right), \ldots,\left(d_{i}, \Omega\left(d_{1}\right)\right)\right\}$.

Proof: Similar to the proof of Lemma 6 , we can prove Corollary 1 through $\varphi\left(\Omega^{\pi \rightarrow \hat{\pi}}, \Omega\right)=\sum_{k=1}^{i} w_{k^{\prime}, k}$.

Corollary 2: If there exists no positive directed loop in $\mathcal{G}^{D}$, the matching $\Omega^{\pi}$ obtained by executing spectrum sharing strategy $\pi$ is a popular matching .

Proof: Based on Lemma 6, since searching for a rotationswap coalition can be equivalent to searching for a positive directed loop, Corollary 2 can be concluded by Definition 5 .

We give a simple example illustrated in Fig. 2, where five active users depicted with solid lines and three virtual users depicted with dashed lines are in three different spectrum sharing groups. Each directed edge only exists between two nodes with different colors. We can find a positive directed loop $d_{2} \rightarrow d_{3} \rightarrow d_{8} \rightarrow d_{1}$. If we want to maintain the popularity of this matching with low complexity, we have to search for a loop involving as many nodes as possible. We invoke the coloring-based heuristic algorithm in [21] to find a directed loop with largest size, for the purpose of maintaining the popularity. The rotation-vote (RV) algorithm to search for a popular matching with externalities can be summarized in Alg.2. Note that (13b), (13d), and (14b) must be satisfied in vote operation.

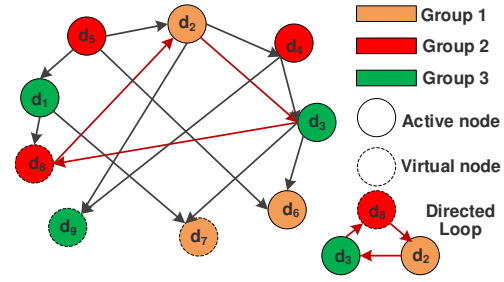

Fig. 2: Illustration of Directed Graph.

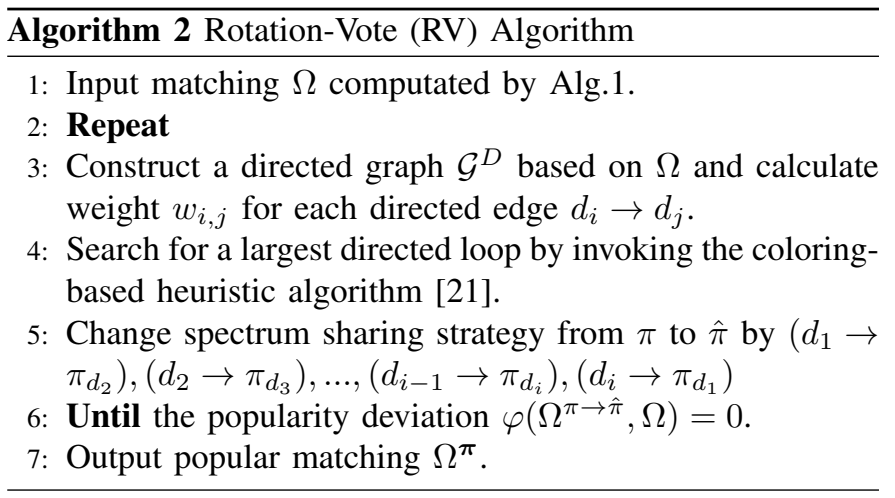

Corollary 3: Alg.2 can converge to popular state within finite iterations.

Proof: Considering that both the vertex set and edge set are finite, the strategic space is finite. Moreover, the popularity can be improved by $\Omega^{\pi \rightarrow \hat{\pi}}=\left\{\left(d_{1}, \Omega\left(d_{2}\right)\right), \ldots,\left(d_{i}, \Omega\left(d_{1}\right)\right)\right\}$ (Corollary 2). Hence, Alg.2 has a bounded iteration.

Computational Complexity of Alg. 2: The complexity for building the graph $\mathcal{G}^{D}(n)$ is $\mathcal{O}\left(\left|\mathcal{E}^{D}(n)\right|+|\widetilde{\mathcal{D}}(n)|\right)$ when the index of iteration is $n$, for searching for the largest loop is $\mathcal{O}\left(\left|\mathcal{E}^{D}(n)\right|+|\widetilde{\mathcal{D}}(n)|\right)$, which can be bounded by $\mathcal{O}\left(M^{2}\right)$. Assume that the iteration number is $\epsilon_{1}$, the complexity taken to compute $\Omega^{\pi \rightarrow \hat{\pi}}$ is $\mathcal{O}\left(\sum_{n=1}^{\epsilon_{1}}\left(\left|\mathcal{E}^{D}(n)\right|+|\widetilde{\mathcal{D}}(n)|\right)\right)$. Note that $\epsilon_{1}$ can be bounded by $C_{N}^{2}$, by considering the worst case that only the loop with only two edges is founded in each iteration and two nodes can exchange their groups at most two times. Thus, the complexity in the worst case can be bounded by $\mathcal{O}\left((N M)^{2}\right)$, which is much less than that of the solution obtained by Linear Programming, i.e., $\mathcal{O}\left((N M)^{3.5}\right)$ through interior-point method [31], since the iteration number $\epsilon_{1}$ is much less than $C_{N}^{2}$ and the edge number $\left|\mathcal{E}^{D}(n)\right|$ is much less than $M^{2}$ in practice.

Finally, the overall procedure of joint trajectory design, power control, and channel allocation (JTPC) can be summarized as

- Phase 1 (qualification confirmation): Each agent obtains the local CSI and identifies its qualified partner through channel estimation.

- Phase 2 (channel allocation): With the necessary information, each agent invokes Alg.1 to make an initially channel allocation decision. Then, each spectrum sharing group investigates the possible rotation-vote operation through information exchange. One leader $u_{j}$ will negotiate with another leader $u_{j^{\prime}}$ if the utility of its group will improve when one group member $d_{i}$ joins $u_{j}$ 's group. Each leader will record the received request and send this information to those leaders that it negotiates with. Each 
leader can keep a label to show its state: 1) has not been negotiated with; 2) has been negotiated with; or 3) has been involved in a loop. The rotation-vote request will be broadcast when one leader finds a loop through recorded information. Note that the channel allocation decisions are made in each time slot.

- Phase 3 (trajectory design and power control): Each UAV first controls its power through (21) under given trajectory, and then optimize the trajectory by interiorpoint method under given power in a iterative manner.

- Repeat Phase 1-3 until the variables remain unchanged.

Note that JTPC operates in a distributed manner. In the power control and trajectory design stage, the optimization process for UAV is independent i.e., the problem (15) can be decoupled into $M$ sub-problems as (16), and thus each UAV only makes self-organizing decisions to adjust their own power and trajectory through local information. In the channel allocation stage, each user only needs to exchange local information, issues acception/rejection, and negotiates with other user. In both of these two stages, users themselves will make the decisions rather than following the decisions made by the system.

Convergence, Complexity and Signaling Process of JTPC: In the power control and trajectory design stage, given the fixed channel allocation decision $\omega$, the sub-optimal solution is derived and the objective value is non-decreasing. In the channel allocation stage, given the power control and trajectory design decisions $(\boldsymbol{x}, \boldsymbol{y}, \boldsymbol{P})$, the objective value is also non-decreasing. Firstly, when the externalities are not considered, the Alg.1 is proved to obtain the maximal popular matching with finite complexity $\mathcal{O}\left(\left|\mathcal{D}^{\prime}\right| \cdot\left|\mathcal{U}^{\prime}\right|\right)$, which proves its convergence. Secondly, when the externalities are considered, the Corollary 3 proves the convergence of Alg.2 and the objective value is non-decreasing from the unpopular state to the popular state. To sum up, considering that the objective value by optimizing $(\boldsymbol{\omega}, \boldsymbol{x}, \boldsymbol{y}, \boldsymbol{P})$ is non-decreasing over iterations and the solution space is finite, the JTPC can converge to a suboptimal solution. The complexity of Phase 3 is $\mathcal{O}\left(\epsilon_{2} M(4 T+K T)^{3.5}\right)$ [31], where $\epsilon_{2}$ is the iteration number of Phase 3. In summary, the complexity of JTPC is $\mathcal{O}\left(N_{i}\left(\sum_{t=1}^{T} \sum_{n=1}^{\epsilon_{1}(t)}\left(\left|\mathcal{E}^{D}(n)\right|+|\widetilde{\mathcal{D}}(n)|\right)+\epsilon_{2} M(4 T+K T)^{3.5}\right)\right)$ where $N_{i}$ is the iteration number of JTPC.

In phase 1 , the communication overhead consists of CSI at the BS, between UAVs and D2D users, and CSI sharing among D2D users within each group. The CSI information can be shared through the local control channels [10]. We assume that sharing CSI in phase 1 can cost $W_{1}$ bits in messages in one iteration. In the first stage of Phase 2, each D2D user will send one-bit to propose to the desired UAV for spectrum sharing. Then, each UAV decides whether or not accept the request and then respondes with one-bit information. This stage will cost $M N$ bits. In the second stage of Phase 2, each group leader will first negotiate with other leaders and vote for the group member that needed to switch to other groups. Note that the vote considers only group members, i.e., local D2D users and the group leader, i.e., UAV, which generates low communication overhead, i.e., $M^{2} q$ in the worst case by assuming that each quota is fulfilled. Next, each leader will record the received requests and send this information to those leaders that it negotiates with, which also generates low communication overhead, i.e, $M N W_{2}$ by assuming that every two nodes have negotiation and send at most $W_{2}$ bits messages. The final channel allocation results can be obtained when the largest loop is found. In phase 3, no information exchange is needed since each UAV optimizes its power and trajectory under given information. Hence, the overall signaling overhead of JTPC is $T N_{i}\left(W_{1}+M N+\epsilon_{1}\left(M^{2} q+M N W_{2}\right)\right)$, which is acceptable for practical implementation.

Based on the above discussion, the solution derived by JTPC is local optimal. Firstly, in the power control and trajectory design stage, the sub-optimal solution can be derived. In the channel allocation stage, the stable solution obtained by matching theory is local optimal and can strike the tradeoff performance between (13) and (14). Hence, our proposed algorithms can achieve the local optimal solution with the guarantee of computational complexity.

\section{Simulation Results and Performance Analysis}

In order to demonstrate the validity of our theoretical analysis, we evaluate JTPC regarding the convergence, and trade-off between the average worst-case secrecy rates and achievable rate. We set the parameters based on the existing works [6], [13], [14], [17], which are applicable for UAV communication of most heterogeneous cellular networks. Generally, the parameters are set as follows unless being specified otherwise. The network area is $\left(1 \times 1 \times 1 \mathrm{~km}^{3}\right)$, where the number of UAVs is 8 and that of D2D users is 50, respectively. The BS is located in the center. The D2D pairs' locations are uniformly distributed within the coverage. The distance between D2D transmitter and receiver is no more than $50 \mathrm{~m}$. The number of time slots is 160 and the duration $\Delta=1 \mathrm{~s}$. In each slot $t$, each UAV flies to the location near BS $b$ and its maximum speed $v_{\max }=10 \mathrm{~m} / \mathrm{s}$. In this paper, we assume that there are four locations, whose coordinates are as follows: location 1 (-400, -400), location 2 (400, -400), location 3 (400, 400), and location $4(-400,400)$. Synchronously, every two UAVs fly from location $n$ to $(n \bmod 4)+1, n \in[1,4]$. Given the , predefined path, if one UAV does not have sufficient time to reach the location of $b$, it will directly fly to the final location. Besides, there are $K=8$ eavesdroppers, whose estimated location are uniformly distributed within the coverage. The radius $\mathcal{R}_{k}$ is uniformly distributed within the range $20 \sim 80 \mathrm{~m}$. The hovering altitude is uniformly distributed within the range $80 \sim 120 \mathrm{~m}$. The transmit power of terrestrial devices is 10 $\mathrm{dBm} . \bar{P}_{j}=20 \mathrm{dBm}$ and $\hat{P}_{j}=4 \bar{P}_{j}$. The path loss parameter for $\mathrm{G} 2 \mathrm{G}$ links $\alpha_{G}$ is set to 3 . The power gain $d_{0}=1 \mathrm{~m}$ is set to $\eta_{0}=-60 \mathrm{~dB}$. The noise power $N_{0}$ is $-120 \mathrm{dBm}$. The quota $q$ for each UAV is fixed at 5. The mutual interference threshold for UAVs is $-90 \mathrm{~dB}$ and secrecy rate threshold for involved D2D users is $0.5 \mathrm{bps} / \mathrm{Hz}$. In social domain, we consider the Erdos-Renyi social graph with adjustable social link probability $p_{s}=0.6$ as a social graph model [34].

Furthermore, we adopt different benchmark algorithms to comprehensively evaluate our proposed algorithm. Firstly, we 


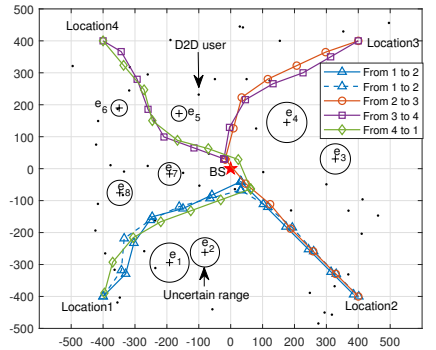

Fig. 3: Trajectories of different UAVs.

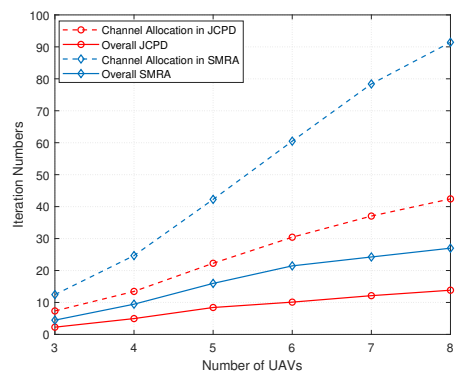

Fig. 4: Iterations versus the number of UAVs.

compare RV with classical stable matching based resource allocation (SMRA) algorithm in [9] and coalition formation based resource allocation (CFRA) algorithm in [10]. Moreover, we provide the performance upper bound achieved by "Exhaustive Search (ES)", whose complexity is very high, i.e., $C_{N}^{M} A_{M}^{M}$ in the best case $(q=1)$. Note that the joint trajectory and power optimization is embedded in these benchmarks. Besides, we invoke ES twice to obtain the global optimums in two respective trade-off performances whilst the others once to analyze the trade-off. Furthermore, we also give the perfermance analysis on the case "fixed power", i.e., equal power allocation over time, and the case "fixed trajectory", where each UAV flies to the location right above BS at its maximal speed, then hovers over the BS for as long as possible, and finally reaches the final location at time $T$.

Fig. 3 shows the trajectories of different UAVs. To avoid the confusion caused by trajectory intersection, we only choose capture the trajectories of 5 UAVs. For the trajectory from location 1 to 2, although two UAVs take off at the same time, their trajectories are different due to their different altitudes and channel selection decisions. The commonality is that both two UAVs first fly in an arc path to bypass the eavesdroppers $e_{1}, e_{2}, e_{7}$, and $e_{8}$, and reach a certain point near BS; then they hover at that point for as long as possible, and finally fly to the final location. It is worth mentioning that the selection of the hover point should consider the distances from the estimated locations of eavesdroppers to BS, the radius of the uncertain region, and the location of those D2D pairs reusing the same channel. Note that the above analysis also applies to the other trajectories.

To evaluate the convergence performance of JTPC, we investigate the iterations needed in channel allocation phase and overall procedure. In Fig. 4, we note that the needed iteration number of JTPC grows with the UAV numbers. It is because

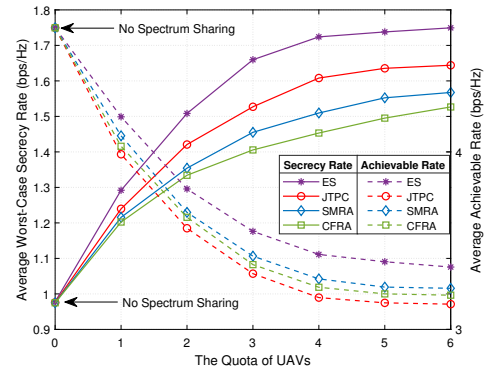

Fig. 5: Trade-off performances of UAVs versus $q$.

the number of nodes will affect the probability of searching for the directed loops. Moreover, Fig. 4 further indicates that the number of iterations for convergence is relatively small compared to that of the total nodes, i.e. $(M+N)$, while the popular matching based method outperforms the stable matching based method in convergence performance.

In Fig. 5, the trade-off performances, i.e., average worstcase secrecy rate and achievable rate, with varying number of quota is shown. Note that the attained performances when $q=0$ represents the case no spectrum sharing is needed, which remain unchanged with the increasing quota. It is obvious that the secrecy rate gradually improves with the increasing quota, since each UAV can recruit more friendly jammers. The eavesdropper learns less information and receives more interference, which improves the secrecy rate. Meanwhile, the achievable rate gradually decreases because more co-channel interferences are introduced. Note that JTPC can achieve the better security performance than SMRA and CFRA with minor loss in achievable rates. The reason behind the superiority is that JTPC can achieve the global stability while SMRA and CFRA can only achieve the local stability. Besides, the maximum popular matching structure can make more D2D users share the cellular spectrum. When $q=5$, the secrecy rates and achievable rates of all algorithms are saturated since the increment in security by recruiting more friendly jammers cannot match the loss in achievable rate and thus the number of recruited D2D users are saturated. Compared with the case of no spectrum sharing, JTPC can achieve approximately $67.5 \%$ increment in secrecy rate with approximately $33 \%$ loss in achievable rate when $q=5$. In Fig. 6, we also analyze the trade-off performances of involved D2D users, i.e., friendly jammers, which show the similar trend with Fig. 5. The differences are as follows: 1) the secrecy rate decreases at $q=5$ due to the severe co-channel interference; and 2) JTPC can achieve the sub-optimal solutions in both security and achievable rate performances.

Next, we further investigate the impact of social trust on security performance. In Fig. 7, we can observe that the secrecy rates gradually improves with the increasing social link probability, because more D2D users become socially trusted and then join in the sepctrum sharing groups. It is worth mentioning that a stage arrives when the secrecy rates reach a saturation region, with further increase of social link probability. Because the increment in security by recruiting more socially-trusted jammers cannot match the loss in achievable rate. The case $p_{s}>0.6$ can ensure that most D2D users join 


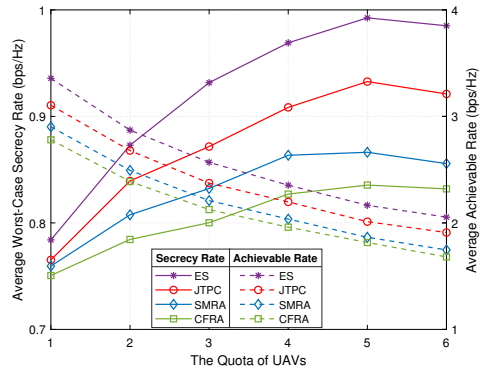

Fig. 6: Trade-off performances of D2D users versus $q$.

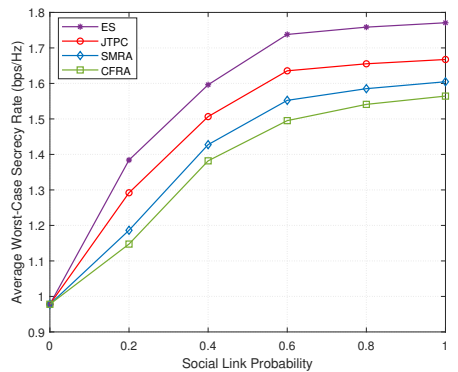

Fig. 7: Average worst-case secrecy rate of UAVs versus $p_{s}$.

in spectrum sharing. Fig. 8 shows the security performance of JTPC versus the duration $T$ and average power $\bar{P}$, respectively. We can observe that the secrecy rates increase with both $T$ and $\bar{P}$ whilst reach a saturation region when $\bar{P}$ is high enough, because the problem (16) is independent of the power $P_{j}(t)$ and determined by $\left(x_{j}(t), y_{j}(t)\right)$ when $P_{j}(t)$ is high enough. Note that $T \geq 80$ can guarantee that each UAV can fly from the initial location to the final location. Compared with the cases of "fix trajectory" and "fix power", JTPC can achieve approximately $18.1 \%$ and $15.6 \%$ increments in secrecy rate, respectively, when other parameters remain unchanged as in the initial setting.

Through comparison, JTPC can achieve the better tradeoff between security and achievable rate performances with acceptable computational complexity.

\section{CONCLUSION}

In this paper, we proposed the concept of SIoFT, by leveraging inter-device social trust to enhance the security. Joint trajectory design, power control, and channel allocation method has been proposed to optimize the average worst-case secrecy rate of $\mathrm{A} 2 \mathrm{G}$ communication whilst the locations of eavesdroppers cannot be perfectly known. Simulation results demonstrated the superiority of popular matching model on different performance metrics. We believe that the initial model and algorithm proposed here have potential to achieve the global stability for the resource allocation problems in other wireless communication scenarios.

\section{REFERENCES}

[1] M. R. Palattella, M. Dohler, A. Grieco, G. Rizzo, J. Torsner, T. Engel, and L. Ladid, "Internet of things in the 5G era: Enablers, architecture, and business models," IEEE J. Sel. Areas Commun., vol. 34, pp. 510527, Mar. 2016.

[2] L. Atzori, A. Iera, G. Morabito, and M. Nitti, "The Social Internet of Things (SIoT) - when social networks meet the Internet of Things: Concept, architecture and network characterization," Comput. Netw., vol. 56, pp. 3594-3608, Nov. 2012.

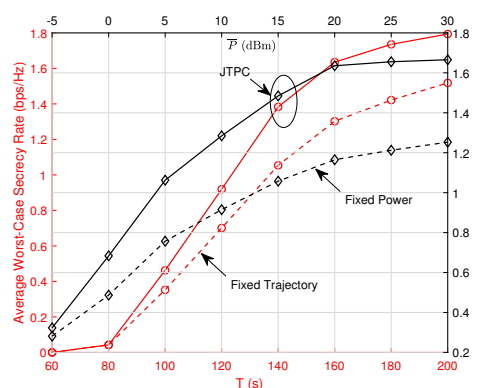

Fig. 8: Average worst-case secrecy rate versus $T$ and $\bar{P}$.

[3] I. Bekmezci, O. K. Sahingoz, and S. Temel, "Flying ad-hoc networks (fanets): A survey," Ad Hoc Netw., vol. 11, pp. 1254 - 1270, May. 2013.

[4] D. F. Pigatto, M. Rodrigues, J. V. de Carvalho Fontes, A. S. R. Pinto, J. Smith, and K. R. L. J. C. Branco, The Internet of Flying Things, ch. 19, pp. 529-562. John Wiley \& Sons, Ltd, 2018.

[5] S. W. Loke, "The internet of flying-things: Opportunities and challenges with airborne fog computing and mobile cloud in the clouds," CoRR, vol. abs/1507.04492, Jul. 2015.

[6] M. Mozaffari, W. Saad, M. Bennis, and M. Debbah, "Unmanned aerial vehicle with underlaid device-to-device communications: Performance and tradeoffs," IEEE Trans. Wireless Commun., vol. 15, pp. 3949-3963, Jun. 2016.

[7] Q. Wu, W. Mei, and R. Zhang, "Safeguarding wireless network with UAVs: A physical layer security perspective," IEEE Wireless Commun., vol. 26, pp. 12-18, Oct. 2019.

[8] S. Leung-Yan-Cheong and M. Hellman, "The Gaussian wire-tap channel," IEEE Trans. Inform. Theory, vol. 24, pp. 451-456, Jul. 1978.

[9] X. Chen, Y. Zhao, Y. Li, X. Chen, N. Ge, and S. Chen, "Social trust aided D2D communications: Performance bound and implementation mechanism,” IEEE J. Sel. Areas Commun., vol. 36, pp. 1593-1608, Jul. 2018.

[10] M. Ahmed, Y. Li, Z. Yinxiao, M. Sheraz, D. Xu, and D. Jin, "Secrecy ensured socially aware resource allocation in device-to-device communications underlaying HetNet," IEEE Trans. Veh. Technol., vol. 68, pp. 4933-4948, May. 2019.

[11] X. Chen, B. Proulx, X. Gong, and J. Zhang, "Exploiting social ties for cooperative D2D communications: A mobile social networking case," IEEE/ACM Trans. Netw., vol. 23, pp. 1471-1484, Oct. 2015.

[12] M. Waqas, M. Ahmed, Y. Li, D. Jin, and S. Chen, "Social-aware secret key generation for secure device-to-device communication via trusted and non-trusted relays," IEEE Trans. Wireless Commun., vol. 17, pp. 3918-3930, Jun. 2018.

[13] G. Zhang, Q. Wu, M. Cui, and R. Zhang, "Securing UAV communications via joint trajectory and power control," IEEE Trans. Wireless Commun., vol. 18, pp. 1376-1389, Feb. 2019.

[14] M. Cui, G. Zhang, Q. Wu, and D. W. K. Ng, "Robust trajectory and transmit power design for secure UAV communications," IEEE Trans. on Veh. Technol., vol. 67, pp. 9042-9046, Sep. 2018.

[15] H. Kang, J. Joung, J. Ahn, and J. Kang, "Secrecy-aware altitude optimization for quasi-static UAV base station without eavesdropper location information," IEEE Commun. Letters, vol. 23, pp. 851-854, May. 2019.

[16] H. Zhang, T. Wang, L. Song, and Z. Han, "Radio resource allocation for physical-layer security in D2D underlay communications," in Proc. IEEE ICC, (Sydney, NSW, Australia), pp. 2319-2324, Jun. 2014.

[17] K. Zhang, M. Peng, P. Zhang, and X. Li, "Secrecy-optimized resource allocation for device-to-device communication underlaying heterogeneous networks," IEEE Trans. Veh. Technol., vol. 66, pp. 1822-1834, Feb. 2017.

[18] Y. Gu, W. Saad, M. Bennis, M. Debbah, and Z. Han, "Matching theory for future wireless networks: fundamentals and applications," IEEE Commun. Mag., vol. 53, pp. 52-59, May. 2015.

[19] W. Saad, Z. Han, T. Basar, M. Debbah, and A. Hjorungnes, "A selfish approach to coalition formation among unmanned air vehicles in wireless networks," in Proc. IEEE GAMENETS, (Istanbul, Turkey), pp. 259-267, May. 2009.

[20] B. Wang, Y. Sun, Z. Sheng, H. M. Nguyen, and T. Q. Duong, "Inconspicuous manipulation for social-aware relay selection in flying internet of things," IEEE Wireless Commun. Letters, vol. 8, pp. 1394-1397, Oct. 2019. 
[21] B. Wang, Y. Sun, S. Li, and Q. Cao, "Hierarchical matching with peer effect for low-latency and high-reliable caching in social IoT," IEEE Internet Things J., vol. 6, pp. 1193-1209, Feb. 2019.

[22] K. Hamidouche, W. Saad, and M. Debbah, "Popular matching games for correlation-aware resource allocation in the internet of things," in Proc. IEEE GLOBECOM, (Singapore), pp. 1-6, Dec. 2017.

[23] D. Gale and L. S. Shapley, "College admissions and the stability of marriage," AM. Math. Monthly, vol. 69, pp. 9-15, Jan. 1962.

[24] D. Abraham, R. Irving, T. Kavitha, and K. Mehlhorn, "Popular matchings," SIAM J. Comput., vol. 37, pp. 1030-1045, Sep. 2007.

[25] P. Biró, R. W. Irving, and D. F. Manlove, "Popular matchings in the marriage and roommates problems," in Proc. CIAC (T. Calamoneri and J. Diaz, eds.), (Berlin, Heidelberg), pp. 97-108, Springer Berlin Heidelberg, May. 2010.

[26] T. Kavitha, "A size-popularity tradeoff in the stable marriage problem," SIAM J. Comput., vol. 43, no. 1, pp. 52-71, 2014

[27] S. Bayat, R. H. Y. Louie, Z. Han, B. Vucetic, and Y. Li, "Physical-layer security in distributed wireless networks using matching theory," IEEE Trans. Inf. Foren. Sec., vol. 8, pp. 717-732, May. 2013.

[28] S. Coleri, M. Ergen, A. Puri, and A. Bahai, "Channel estimation techniques based on pilot arrangement in OFDM systems," IEEE Trans. Broadcast., vol. 48, pp. 223-229, Sep. 2002.

[29] Y. Wang, Z. Miao, R. Sun, and L. Jiao, "Distributed coalitional game for friendly jammer selection in ultra-dense networks," EURASIP J. Wirel. Comm., vol. 2016, pp. 2-11, Sep. 2016.

[30] X. Lin, V. Yajnanarayana, S. D. Muruganathan, S. Gao, H. Asplund, H. Maattanen, M. Bergstrom, S. Euler, and Y. P. E. Wang, "The sky is not the limit: LTE for unmanned aerial vehicles," IEEE Commun. Mag., vol. 56, pp. 204-210, Apr. 2018.

[31] S. Boyd and L. Vandenberghe, Convex Optimization. New York, NY, USA: Cambridge University Press, 2004.

[32] R. J. Wilson, Introduction to Graph Theory. New York, NY, USA: John Wiley \& Sons, Inc., 1986.

[33] F. Guo, H. Lu, D. Zhu, and H. Wu, "Interference-aware user grouping strategy in NOMA systems with QoS constraints," in Proc. IEEE INFOCOM, (Paris, France), 2019.

[34] M. E. Newman, D. J. Watts, and S. H. Strogatz, "Random graph models of social networks," Proc. Natl. Acad. Sci. USA, vol. 99 Suppl 1, pp. 2566-2572, Feb. 2002.

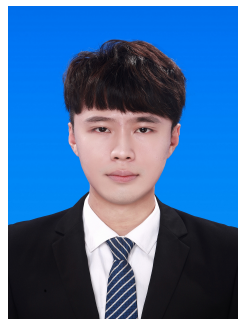

Bowen Wang received the Ph.D. degree in information and communication engineering from China University of Mining and Technology, Xuzhou, China, in 2020. He was also a visiting Ph.D student at the School of Electronics, Electrical Engineering and Computer Science, Queen's University Belfast from 2018 to 2019. He is currently an Associate Professor at the School of Information and Control Engineering, China University of Mining and Technology. He was awarded the best paper award in INISCOM 2019. His research interests include matching theory, graph theory, UAV communications, and Social Internet of Things (SIoT).

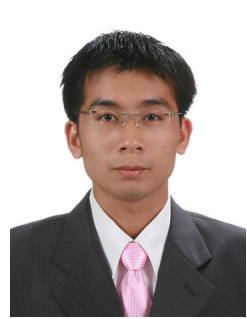

Trung Q. Duong (S'05, M'12, SM'13) received his $\mathrm{Ph} . \mathrm{D}$. degree in Telecommunications Systems from Blekinge Institute of Technology (BTH), Sweden in 2012. Currently, he is with Queen's University Belfast (UK), where he was a Lecturer (Assistant Professor) from 2013 to 2017 and a Reader (Associate Professor) from 2018. His current research interests include Internet of Things (IoT), wireless communications, molecular communications, and signal processing. He is the author or co-author of 290 technical papers published in scientific journals (165 articles) and presented at international conferences (125 papers).

Dr. Duong currently serves as an Editor for the IEEE TRANSACTIONS ON WIRELESS COMMUNICATIONS, IEEE TRANSACTIONS ON COMMUNICATIONS, IET COMMUNiCATIONS, and a Lead Senior Editor for IEEE COMMUNiCATIONS LetTERs. He was awarded the Best Paper Award at the IEEE Vehicular Technology Conference (VTC-Spring) in 2013, IEEE International Conference on Communications (ICC) 2014, IEEE Global Communications Conference (GLOBECOM) 2016, and IEEE Digital Signal Processing Conference (DSP) 2017. He is the recipient of prestigious Royal Academy of Engineering Research Fellowship (2016-2021) and has won a prestigious Newton Prize 2017

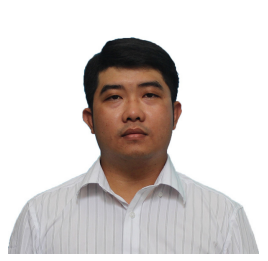

Long D. Nguyen received his B.S. degree in Electri$\mathrm{cal}$ and Electronics Engineering and M.S. degree in Telecommunication Engineering from Ho Chi Minh City University of Technology (HCMUT), Vietnam, in 2013 and 2015, respectively. He received his $\mathrm{Ph} . \mathrm{D}$. degree in Electronics and Electrical Engineering from Queen s Univerisity Belfast (QUB), UK, in 2018. He is currently with Duy Tan University as an Adjunct Assistant Professor and Dong Nai University in Vietnam as an Assistant Professor. Dr. Nguyen was awarded the Best Paper Award at the IEEE Digital Signal Processing (DSP) 2017, the IEEE International Conference on Recent Advances in Signal Processing, Telecommunication and Computing (Sigtelcom) 2018, the IEEE International Conference on Communications (ICC) 2019, the International Wireless Communications \& Mobile Computing Conference (IWCMC) 2019 and the IEEE Global Communications Conference (GLOBECOM) 2019. He was also awarded the Exemplary Reviewer Award in IEEE Communications Letters 2018.

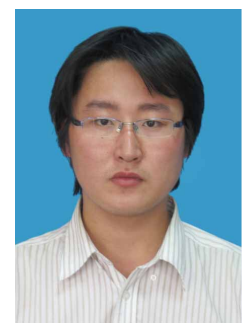

Nan Zhao (S'08-M'11-SM'16) is currently a Professor at Dalian University of Technology, China. $\mathrm{He}$ received the Ph.D. degree in information and communication engineering in 2011, from Harbin Institute of Technology, Harbin, China.

Dr. Zhao is serving on the editorial boards of IEEE Wireless Communications, IEEE Wireless Communications Letters and IEEE Transactions on Green Communications and Networking. He won the best paper awards in IEEE VTC 2017 Spring, ICNC 2018, WCSP 2018 and WCSP 2019. He also received the IEEE Communications Society Asia Pacific Board Outstanding Young Researcher Award in 2018. 